\title{
The critical merger distance between two co-rotating quasi-geostrophic vortices
}

\author{
By JEAN N. REINAUD AND DAVID G. DRITSCHEL \\ Mathematical Institute, University of St Andrews, North Haugh, St Andrews KY16 9SS, UK
}

(Received 14 July 2003 and in revised form 28 May 2004)

This paper examines the critical merger or strong interaction distance between two equal-potential-vorticity quasi-geostrophic vortices. The interaction between the two vortices depends on five parameters: their volume ratio, their height-to-width aspect ratios and their vertical and horizontal offsets. Due to the size of the parameter space, a direct investigation solving the full quasi-geostrophic equations is impossible. We instead determine the critical merger distance approximately using an asymptotic approach. We associate the merger distance with the margin of stability for a family of equilibrium states having prescribed aspect and volume ratios, and vertical offset. The equilibrium states are obtained using an asymptotic solution method which models vortices by ellipsoids. The margin itself is determined by a linear stability analysis. We focus on the interaction between oblate to moderately prolate vortices, the shapes most commonly found in turbulence. Here, a new unexpected instability is found and discussed for prolate vortices which is manifested by the tilting of vortices toward each other. It implies than tall vortices may merge starting from greater separation distances than previously thought.

\section{Introduction}

Vortical structures abound in the Earth's atmosphere and oceans. Vortices - or coherent volumes of anomalous potential vorticity (PV) - are known to play an important dynamical role in these environments, cf. Holton et al. (1995), and Garrett (2000). Their behaviour is strongly influenced by both stratification and the Earth's rotation, and is fundamentally distinct from the motion of higher-frequency inertiagravity waves. Vortices belong to the dominant, 'balanced' class of fluid motions that depends only on the PV distribution, cf. Hoskins, McIntyre \& Robertson (1985).

Vortices induce fluid motion and sometimes strongly interact as in 'merger'. However, other forms of strong interaction are frequently observed, such as partial merger, in which part or parts of the smaller vortex do not merge with the larger vortex, cf. Dritschel (2002). Hereinafter, 'merger' will refer to any form of strong interaction which results in a significant change in volume of one of the vortices.

Vortex merger has been extensively studied in the two-dimensional context, for it provides a scenario to explain physically the average 'inverse energy cascade' and the 'direct enstrophy cascade' observed in two-dimensional turbulence. Energy is said to move to larger scales as a result of the formation of a few larger vortices, while low-energy small vortices and filaments generated during the merger process feed the small scales of the enstrophy spectrum.

Studies of two-dimensional vortex merger have been conducted using both direct numerical simulation (e.g. see Waugh 1992 and references therein), and linear stability analyses, see Dritschel (1995) and Meunier et al. (2002). In linear stability analyses, 
the critical distance for vortex merger is associated with the margin of stability for two vortices in mutual equilibrium. The 'exchange-type' instability observed in these studies has been shown to coincide with local extrema in both the angular impulse and the 'excess' energy of the flow as a function of the separation distance between the vortices, see Dritschel (1995) for uniform-PV vortices and Meunier et al. (2002) for distributed vortices. A theoretical justification, based on Kelvin's variational principle is proposed in Saffman (1992).

Direct enstrophy and inverse energy cascades are also observed in three-dimensional quasi-geostrophic turbulence, see Hua \& Haidvogel (1986). The quasi-geostrophic (QG) model is the simplest model containing the dominant features of stable stratification and background rotation found in the oceans and the atmosphere. The quasi-geostrophic approximation gives a single dynamical equation for the evolution of the PV anomaly (hereinafter referred to as PV, for simplicity). The anomaly represents the departure of the full PV field from the background PV distribution associated with the Earth's rotation. While the PV distribution is fundamentally threedimensional, the motion is constrained to remain parallel to stratification surfaces, and this gives rise to a layer-wise two-dimensional flow. Moreover, in the absence of dissipative and diabatic effects, the PV is a Lagrangian invariant. That is, the PV is conserved following each fluid element.

Recently, vortex merger in quasi-geostrophic flows has been studied by von Hardenberg et al. (2000), Dritschel (2002) and Reinaud \& Dritschel (2002). In von Hardenberg et al. (2000) and in Dritschel (2002), the focus was on the influence of the vortex height-to-width aspect ratio on the merger distance between equal-volume equal-PV (or equal-distributed-PV) horizontally aligned vortices. It was concluded in Dritschel (2002) that the merger of tall vortices does not tend to the limiting case of barotropic two-dimensional vortices since vortices are inclined to be destabilized three-dimensionally - or baroclinically - by the 'tall column instability', analysed in Dritschel \& de la Torre Juárez (1996). On the other hand, Reinaud \& Dritschel (2002) focused on the effect of the vertical offset on the merger of unit-aspect-ratio vortices to investigate the fundamental anisotropy of the QG equations (i.e. the lack of vertical advection). Again, in that study the vortices had the same volume and PV. It was found that vortices moderately offset in the vertical merge from a greater separation distance than do vortices not offset vertically. This result can be explained by the different roles played by horizontal strain and vertical shear - the latter appearing to be more destructive as a consequence of the lack of vertical advection. In that work, the margin of stability was shown to coincide with the local minimum of the angular impulse and the maximum of the energy as a function of the horizontal 'gap'; the horizontal gap corresponds to the horizontal distance between the two innermost edges of the vortices.

Nonetheless, little is known about merger in general. For instance, we do not know how vortices of unequal volume offset in the vertical interact. Nor do we know how vortices of different aspect ratio interact. The limited data presently available cannot be extrapolated to understand vortex interactions in general. Our motivation is to fill this gap in understanding and to provide a comprehensice picture.

Vortex interactions depend on five essential parameters even in the case studied here of uniform equal PV. These parameters are the volume ratio of the two vortices, their height-to-width aspect ratios, and their vertical and horizontal offsets. The comprehensive study of such a large parameter space using direct numerical simulation or a full stability analysis as performed in Dritschel (2002) and in Reinaud \& Dritschel (2002) is simply beyond reach. One needs to make an approximation. 
The approximation used here employs the 'quasi-geostrophic ellipsoidal vortex model' (ELM) introduced in Dritschel, Reinaud \& McKiver (2004), which represents vortices as PV ellipsoids and filters higher-order non-ellipsoidal deformations. Interacting ellipsoids (especially in gravitational studies) have been studied by numerous mathematicians starting from MacLaurin (1742) and Laplace (1784), see the reviews by Todhunter (1873) and Chandrasekar (1969). The results have been more recently adapted to the quasi-geostrophic fluid equations by Meacham (1992), Meacham et al. (1994) and Meacham, Morisson \& Flierl (1997) where the motion of a PV ellipsoid in a linear background flow was considered. McKiver \& Dritschel (2003) revisited the topic, proposing a new set of prognostic variables which are used in the ELM. Note that in an independent effort Hashimoto, Shimonishi \& Miyazaki (1999), Miyazaki, Ueno \& Shimonishi (1999) and Miyazaki, Furuichi \& Takahashi (2001) proposed a 'moment expansion ellipsoidal model' to study the interaction between ellipsoids. Our own dynamical approach differs from theirs. It does not rely on second-order moment expansions, and as a result it provides higher accuracy.

The ELM greatly simplifies the QG dynamical problem since a vortex is fully described by its centroid position and a $3 \times 3$ 'shape'-matrix in which only six variable coefficients are independent. More than this, among these nine degrees of freedom, only seven are time dependent. The ELM formalism is here adapted to find families of equilibrium states (or relative equilibria in an appropriate co-rotating frame). A family is characterized by the vertical offset between the two vortices, their volume ratio and their respective aspect ratios. Members of the family differ only in their horizontal gap. We obtain the equilibria using an iterative method similar to that developed for a single vortex in a linear background flow by Reinaud, Dritschel \& Koudella (2003). The equilibrium states are then subjected to a linear stability analysis based on the ELM.

In the present study, we limit the investigation to oblate and moderately prolate vortices - we do not consider vortices having an aspect ratio $h / r$ greater than 1.6. Here $h$ is the half-height of the vortex and $r$ is its mean horizontal radius. This is motivated by the results of Reinaud et al. (2003) (in particular their figure 4) showing that taller vortices are rare in QG turbulence. They represent only $2.5 \%$ of the entire population of vortices.

The paper is organized as follows. Section 2 briefly reviews the QG equations and the ELM model. Then, the numerical methods adapted from the ELM to compute the steady states and then linear stability are described. Section 3 compares the ELM results and results previously obtained in Reinaud \& Dritschel (2002) using the full QG equations. We go on to discuss the full parameter space, in particular the properties of the marginally unstable equilibria, including the critical merger distance. A surprise is found for prolate vortices, namely a new tilting instability, which is present even for symmetric horizontally aligned vortices. This instability is linked to a symmetrybreaking bifurcation to a branch of unstable asymmetric equilibria. Most significantly, it is the first instability encountered when decreasing the vortex separation, and had not been noticed in previous studies due to the imposed symmetry. Finally, our conclusions are offered in $\S 4$.

\section{The quasi-geostrophic ellipsoidal model}

\subsection{The quasi-geostrophic equations}

The inviscid quasi-geostrophic model results from an asymptotic expansion of Euler's equations for $\epsilon=H / L \ll 1, H$ and $L$ being characteristic vertical and horizontal 
length scales, and for $F r^{2} \ll R o \ll 1$ where $F r$ and $R o$ are respectively the Froude and Rossby numbers, see e.g. Gill (1982) for a complete discussion. Following numerous previous studies we consider a constant buoyancy frequency, denoted $N$, and a constant Coriolis frequency, denoted $f$.

In rescaled coordinates in which the vertical coordinate is stretched by Prandtl's ratio $N / f$, the governing equations reduce to

$$
\begin{gathered}
\frac{\mathrm{D} q}{\mathrm{D} t}=0, \\
\Delta \psi=q, \\
\boldsymbol{u}=\mathscr{L} \nabla \psi,
\end{gathered}
$$

where $q(x, y, z, t)$ is the $\mathrm{PV}, \psi$ is the streamfunction, and $\boldsymbol{u}=(u, v)$ is the horizontal velocity. In (2.1), $\mathrm{D} / \mathrm{D} t=\partial / \partial t+u \partial / \partial x+v \partial / \partial y$ stands the material derivative; while in (2.2) $\Delta$ is the three-dimensional Laplace operator, and finally

$$
\mathscr{L}=\left(\begin{array}{ccc}
0 & -1 & 0 \\
1 & 0 & 0 \\
0 & 0 & 0
\end{array}\right)
$$

We do not consider here (generally weak) dissipative or diabatic effects. Hence, in equation (2.1) the PV is materially conserved. Moreover, the PV is constrained to layer-wise two-dimensional motion tangent to stratification surfaces, i.e. $w=0$.

\subsection{Formulation of the model}

The ELM, described in detail in Dritschel et al. (2004) is a finite Hamiltonian system for the motion and the deformation of interacting fluid ellipsoids. For the $i$ th ellipsoid the governing equations are

$$
\begin{aligned}
\frac{\mathrm{d} \boldsymbol{X}_{i}}{\mathrm{~d} t} & =-\frac{1}{\kappa_{i}} \mathscr{L} \frac{\partial H}{\partial \boldsymbol{X}_{i}}, \\
\frac{\mathrm{d} \mathscr{B}_{i}}{\mathrm{~d} t} & =\mathscr{S}_{i} \mathscr{B}_{i}+\mathscr{B}_{i} \mathscr{S}_{i}^{T}, \\
\mathscr{S}_{i} & =-\frac{10}{\kappa_{i}} \mathscr{L} \frac{\partial H}{\partial \mathscr{B}_{i}},
\end{aligned}
$$

where $\boldsymbol{X}_{i}=\left(X_{i}, Y_{i}, Z_{i}\right)$ is the centroid position, and $\mathscr{B}_{i}$ denotes the $3 \times 3$ symmetric matrix in terms of which the boundary of the ellipsoid is expressed by $\left(\boldsymbol{x}-\boldsymbol{X}_{i}\right)^{T} \mathscr{B}_{i}^{-1}\left(\boldsymbol{x}-\boldsymbol{X}_{i}\right)=1$. Also, $\kappa_{i}$ is the 'strength' of vortex $i$, i.e. the volume integral of PV divided by $4 \pi$ (denoting $V_{i}$ the volume of the $i$ th vortex, we have $\kappa_{i}=q_{i} V_{i} / 4 \pi$ for uniform-PV ellipsoids). $H$ is the Hamiltonian of the system, namely the total energy of the system divided by $4 \pi$. Finally, $\mathscr{S}_{i}$ is the flow matrix of the locally linear velocity field at the boundary of the $i$ th ellipsoid: $\boldsymbol{u}(\boldsymbol{x}) \simeq \boldsymbol{u}\left(\boldsymbol{X}_{i}\right)+\mathscr{S}_{i}\left(\boldsymbol{x}-\boldsymbol{X}_{i}\right)$. The assumption that the velocity field is locally linear is needed to ensure that vortices remain ellipsoids, i.e. it filters non-ellipsoidal deformations. In practice, $\mathscr{S}_{i}$ can be decomposed as $\mathscr{S}_{i}=\mathscr{S}_{i}^{v}+\mathscr{S}_{i}^{b}$ where $\mathscr{S}_{i}^{v}$ is the self-induced part and $\mathscr{S}_{i}^{b}$ is the part induced by all other ellipsoids in the flow. The self-induced flow is exactly linear at the boundary of the ellipsoid (see e.g. Chandrasekar 1969) although this is only approximately true for the part induced by other ellipsoids. $\mathscr{S}_{i}^{v}$ is well known and can be evaluated in terms of elliptic integrals of the second kind (Chandrasekar 1969).

The interaction part $\mathscr{S}_{i}^{b}$ is computed approximately by modelling each ellipsoid by a finite sum of singularities. The locations and strengths of the singularities are 
obtained in such a way that the approximate streamfunction deduced from these singularities (point vortices) matches the exact one at a prescribed order of accuracy in $1 / d$, where $d$ is the distance between the vortex centroid and the evaluation point. For example, using seven point vortices, the error in the streamfunction is only of $O\left(1 / d^{7}\right)$. The singularities are used to efficiently compute the interaction energy and consequently the interaction Hamiltonian $H^{b}$. The interaction Hamiltonian $H^{b}$ corresponds to the Hamiltonian of a system of point vortices although these point vortices are not free to move but are attached to the ellipsoids. The flow matrix $\mathscr{S}^{b}$ is then derived explicitly from $H^{b}$. In this study, we use seven singularities to model the external flow induced by a given ellipsoid. Full details concerning the accuracy of the model are addressed in Dritschel et al. (2004).

\subsection{The steady states}

We now present the numerical approach used to compute the steady, equilibrium states. These are relative equilibria, i.e. with respect to a steadily rotating reference frame. The method used here is developed from the approach described in Reinaud et al. (2003), where cases of a single equilibrium ellipsoid in a linear background flow were investigated. The algorithm aims to solve the nonlinear set of equations

$$
\mathscr{S}_{i} \mathscr{B}_{i}+\mathscr{B}_{i} \mathscr{S}_{i}^{T}=0, \quad i=1,2,
$$

in the rotating reference frame where $\mathrm{d} \boldsymbol{X}_{i} / \mathrm{d} t=0$. The volume and the mean aspect ratio of each vortex are prescribed together with the 'gap' between the two vortices. This gap is the horizontal distance between the innermost edges of the vortices. The vortices are aligned along the $x$-axis (their centres lie in the $y, z$-plane). The gap $\delta$ therefore can be expressed in terms of the centroid separation and the coefficients of the $\mathscr{B}$ matrix as

$$
\delta=\left|X_{1}-X_{2}\right|-\sqrt{\left(\mathscr{B}_{1}\right)_{11}}-\sqrt{\left(\mathscr{B}_{2}\right)_{11}}
$$

The gap is used instead of $X_{1}-X_{2}$ because the equilibrium states turn out not to be monotonic in $X_{1}-X_{2}$.

We solve this nonlinear problem using a linear iterative method. Starting from a guess for the equilibrium state $\left(\boldsymbol{X}_{i}^{0}, \mathscr{B}_{i}^{0}\right)_{i=1,2}$, we first calculate the velocities of the vortex centroids, in the reference frame anchored at the global centroid $\boldsymbol{x}_{c}$ of the two vortices, which is the centre of the system rotation:

$$
\boldsymbol{x}_{c}=\frac{\kappa_{1} \boldsymbol{X}_{1}+\kappa_{2} \boldsymbol{X}_{2}}{\kappa_{1}+\kappa_{2}}=0
$$

This provides us with an estimate of the background rotation rate of the system

$$
\Omega=\frac{1}{2} \sum_{i=1}^{2} \frac{v_{i} X_{i}^{0}-u_{i} Y_{i}^{0}}{\left(X_{i}^{0}\right)^{2}+\left(Y_{i}^{0}\right)^{2}}
$$

where $\boldsymbol{u}_{i} \equiv \mathrm{d} \boldsymbol{X}_{i}^{0} / \mathrm{d} t$. Then, we calculate the corrections $\left(\boldsymbol{X}_{i}^{\prime}, \mathscr{B}_{i}^{\prime}\right)$ to the centroid positions $\boldsymbol{X}_{i}^{0}$ and the matrix coefficients $\mathscr{B}_{i}^{0}$ to reach the equilibrium solution using an ad hoc equation in the reference frame rotating at $\Omega$ :

$$
\left(\mathscr{B}_{i}^{0}+\mathscr{B}_{i}^{\prime}\right)\left(\mathscr{S}_{i}^{T}+\frac{\partial \mathscr{S}_{i}^{T}}{\partial X_{i}} X_{i}^{\prime}+\frac{\partial \mathscr{S}_{i}^{T}}{\partial X_{3-i}} X_{3-i}^{\prime}\right)+\left(\mathscr{S}_{i}+\frac{\partial \mathscr{S}_{i}}{\partial X_{i}} X_{i}^{\prime}+\frac{\partial \mathscr{S}_{i}}{\partial X_{3-i}} X_{3-i}^{\prime}\right)\left(\mathscr{B}_{i}^{0}+\mathscr{B}_{i}^{\prime}\right)=0,
$$


for $i=1$, 2. In these equations, we do not explicitly take into account the terms $\partial \mathscr{S} / \partial \mathscr{B}$ which appear in the full linearization of (2.8), purely for simplicity (and since (2.12) works in practice). Note also that since the vortices are taken to be aligned along the $x$-axis, no $Y_{i}^{\prime}$ corrections need be considered. Moreover, we impose the conservation of the height of the vortex by not correcting the coefficient $(\mathscr{B})_{33}$. To fix the horizontal gap between the two vortices, we linearize (2.9) giving

$$
X_{i}^{\prime}=\frac{\left(\mathscr{B}_{i}^{0}\right)_{11}-\left(X_{i}^{0}-\delta\right)^{2}}{2\left(X_{i}^{0}-\delta\right)}-\frac{\left(\mathscr{B}_{i}^{\prime}\right)_{11}}{2\left(X_{i}^{0}-\delta\right)} .
$$

After dropping the last remaining quadratic terms in (2.12), e.g. terms like

$$
\mathscr{B}_{i}^{\prime} \frac{\partial \mathscr{S}_{i}^{T}}{\partial X_{i}} \frac{\left(\mathscr{B}_{i}^{\prime}\right)_{11}}{2\left(X_{i}^{0}-\delta\right)} \text {, etc. }
$$

we obtain a $10 \times 10$ linear system (for the two $\mathscr{B}$ matrices excluding the $(\mathscr{B})_{33}$ components). This system cannot be inverted however. Two equations are removed and volume conservation is enforced for each vortex. This is done as above by linearizing the determinant of $\left(\mathscr{B}_{i}\right)_{i=1,2}$, since $\operatorname{det}(\mathscr{B})=(3 V / 4 \pi)^{2}$. The iterative procedure is repeated until the r.m.s. correction in the two shape matrices is less than a prescribed tolerance, namely $10^{-12}$ in this study. The initial guess for the first member of a given family is two well-separated spheroids (ellipsoids with circular horizontal cross-sections). This is reasonable since an isolated spheroid is a steady state. Then, once a steady state is found, the horizontal gap $\delta$ is reduced and the iterative procedure is resumed for the next state. The new first guess for this state uses the $\mathscr{B}$ matrices of the previous state.

\subsection{Linear stability analysis}

We next describe the scheme used to calculate the linear stability of the equilibrium states for the two vortices. Let $\left(\overline{\boldsymbol{X}}_{i}, \overline{\mathscr{B}}_{i}\right)_{i=1,2}$ be a steady state, in the relevant rotating reference frame. Since only the relative position of the two vortices matters, we describe the state by $\left(\bar{\ell},\left(\overline{\mathscr{B}}_{i}\right)_{i=1,2}\right)$ where

$$
\ell \equiv X_{1}-X_{2} .
$$

We now superimpose a perturbation $(\tilde{\ell}, \tilde{\mathscr{B}})$ on the steady state. We aim to find the first-order equation for the perturbation. The perturbation itself is chosen to have an exponential dependence on time (the classic eigen form), that is

$$
\begin{aligned}
\mathscr{B} & =\overline{\mathscr{B}}+\tilde{\mathscr{B}}=\overline{\mathscr{B}}+\hat{\mathscr{B}}^{\sigma t}, \\
\hat{\mathscr{B}} & =\left(\begin{array}{ccc}
\hat{B}^{1} & \hat{B}^{2} & \hat{B}^{3} \\
\hat{B}^{2} & \hat{B}^{4} & \hat{B}^{5} \\
\hat{B}^{3} & \hat{B}^{5} & 0
\end{array}\right), \\
\ell & =\left(\ell^{1}, \ell^{2}, \ell^{3}\right)=\bar{\ell}+\tilde{\ell}=\bar{\ell}+\hat{\ell}^{\sigma t}, \\
\hat{\ell} & =\left(\hat{\ell}^{1}, \hat{\ell}^{2}, 0\right),
\end{aligned}
$$

where $\sigma=\sigma_{r}+\mathrm{i} \sigma_{i}$ is a complex number; $\sigma_{i}$ represents the frequency of the mode while $\sigma_{r}$ is the growth rate. Note that both $\hat{\ell}^{3}$ and $\hat{B}^{6}$ are zero, since $\ell^{3}$ and $B^{6}$ are invariant in QG flows. Let $\overline{\mathscr{S}}_{i} \equiv \mathscr{S}\left(\overline{\mathscr{B}}_{i}\right)$, and note that the conditions of equilibrium 
require

$$
\begin{array}{r}
\frac{\mathrm{d} \bar{\ell}}{\mathrm{d} t}=\overline{\boldsymbol{g}}-\Omega \hat{\mathbf{e}}^{3} \times \overline{\boldsymbol{\ell}}=0, \\
\overline{\mathscr{S}}_{i} \overline{\mathscr{B}}_{i}+\overline{\mathscr{B}}_{i} \overline{\mathscr{S}}_{i}^{T}=0, \quad i=1,2,
\end{array}
$$

where $\boldsymbol{g} \equiv \mathrm{d} \boldsymbol{X}_{1} / \mathrm{d} t-\mathrm{d} \boldsymbol{X}_{2} / \mathrm{d} t, \hat{\mathbf{e}}^{3} \equiv(0,0,1)$, and $\mathscr{S}_{i} \equiv \mathscr{S}_{i}^{b}+\mathscr{S}_{i}^{v}-\Omega \mathscr{L}$. Note that both equations are written in the reference frame that steadily rotates with the two vortices. The basic evolution equation for the perturbation $\widetilde{\mathscr{B}}_{i}$, to the first order in perturbation, is

$$
\begin{aligned}
\frac{\mathrm{d} \mathscr{B}_{i}}{\mathrm{~d} t}=\frac{\mathrm{d} \tilde{\mathscr{B}}_{i}}{\mathrm{~d} t}= & \left(\mathscr{S}_{i} \tilde{\mathscr{B}}_{i}+\tilde{\mathscr{B}}_{i} \mathscr{S}_{i}^{T}\right) \\
& +\left(\sum_{k=1}^{2} \frac{\partial \mathscr{S}_{i}^{b}}{\partial \ell^{k}} \tilde{\ell}^{k}+\sum_{k=1}^{5} \frac{\partial\left(\mathscr{S}_{i}^{b}+\mathscr{S}_{i}^{v}\right)}{\partial B_{i}^{k}} \tilde{\boldsymbol{B}}_{i}^{k}+\sum_{k=1}^{5} \frac{\partial \mathscr{S}^{b}}{\partial B_{3-i}^{k}} \tilde{B}_{3-i}^{k}\right) \overline{\mathscr{B}}_{i} \\
& +\overline{\mathscr{B}}_{i}\left(\sum_{k=1}^{2} \frac{\partial\left(\mathscr{S}_{i}^{b}\right)^{T}}{\partial \ell^{k}} \tilde{\ell}^{k}+\sum_{k=1}^{5} \frac{\partial\left(\mathscr{S}_{i}^{b}+\mathscr{S}_{i}^{v}\right)^{T}}{\partial B_{i}^{k}} \tilde{B}_{i}^{k}+\sum_{k=1}^{5} \frac{\partial\left(\mathscr{S}_{i}^{b}\right)^{T}}{\partial B_{3-i}^{k}} \tilde{B}_{3-i}^{k}\right),
\end{aligned}
$$

where the derivatives of $\mathscr{S}$ are hereinafter understood to be evaluated at equilibrium. The linearized equation for the centroid separation is

$$
\frac{\mathrm{d} \ell^{m}}{\mathrm{~d} t}=\frac{\mathrm{d} \tilde{\ell}^{m}}{\mathrm{~d} t}=\sum_{k=1}^{2} \frac{\partial \mathrm{g}^{m}}{\partial \ell^{k}} \tilde{\ell}^{k}+\sum_{k=1}^{5} \frac{\partial \mathrm{g}^{m}}{\partial B_{1}^{k}} \tilde{B}_{1}^{k}+\sum_{k=1}^{5} \frac{\partial \mathrm{g}^{m}}{\partial B_{2}^{k}} \tilde{B}_{2}^{k}-(-1)^{m} \Omega \tilde{\ell}^{3-m} .
$$

All the terms appearing in these equations can be explicitly calculated from the Hamiltonian (cf. Appendix D of Dritschel et al. (2004) where the full expression for $\mathscr{S}$ is provided). Note that $\partial \mathscr{S}_{i}^{b} / \partial B_{i}^{k} \neq 0$. This is due to the fact that the local implicit linearization of the background flow at the boundary of the ellipsoid $i$ depends on its shape, since $H^{b}$ depends on both vortices. The above equations result in a $12 \times 12$ eigenvalue problem. To avoid spurious modes, this is reduced to a $9 \times 9$ problem by imposing, to first order in the preturbation amplitude, conservation of volume (of each vortex) and angular impulse. The linearized constraints on volume give

$$
\begin{aligned}
\left(\bar{B}_{i}^{4} \bar{B}_{i}^{6}-\left(\bar{B}_{i}^{5}\right)^{2}\right) \tilde{B}^{1}+2\left(\bar{B}_{i}^{5} \bar{B}_{i}^{3}-\bar{B}_{i}^{6} \bar{B}_{i}^{2}\right) \tilde{B}^{2}+2\left(\bar{B}_{i}^{5} \bar{B}_{i}^{2}-\bar{B}_{i}^{4} \bar{B}_{i}^{3}\right) \tilde{B}^{3} \\
\quad+\left(\bar{B}_{i}^{1} \bar{B}_{i}^{6}-\left(\bar{B}_{i}^{3}\right)^{2}\right) \tilde{B}^{4}+2\left(\bar{B}_{i}^{2} \bar{B}_{i}^{3}-\bar{B}_{i}^{1} \bar{B}_{i}^{5}\right) \tilde{B}^{5}=0, \quad i=1,2 .
\end{aligned}
$$

The angular impulse $J$ is

$$
J=\iiint q\left(x^{2}+y^{2}\right) \mathrm{d} V
$$

measured in a reference frame anchored at the global centroid of the system of ellipsoids $\boldsymbol{x}_{c}$. The contribution of each ellipsoid to the angular impulse is

$$
J_{i}=4 \pi \kappa_{i}\left(X_{i}^{2}+Y_{i}^{2}+\frac{1}{5}\left(B_{i}^{1}+B_{i}^{4}\right)\right) .
$$

Hence linearized angular impulse conservation implies

$$
2 \kappa_{1} \kappa_{2} \bar{\ell}^{1} \tilde{\ell}^{1}+\frac{1}{5}\left(\kappa_{1}+\kappa_{2}\right)\left[\kappa_{1}\left(\tilde{B}_{1}^{1}+\tilde{B}_{1}^{4}\right)+\kappa_{2}\left(\tilde{B}_{2}^{1}+\tilde{B}_{2}^{4}\right)\right]=0 .
$$

In practice, we remove the equation for $\mathrm{d} \tilde{\ell}^{1} / \mathrm{d} t$ and the two equations for $\mathrm{d} \tilde{B}_{i}^{1} / \mathrm{d} t, i=1,2$ from the system. Note also that in the derivation of the method we 


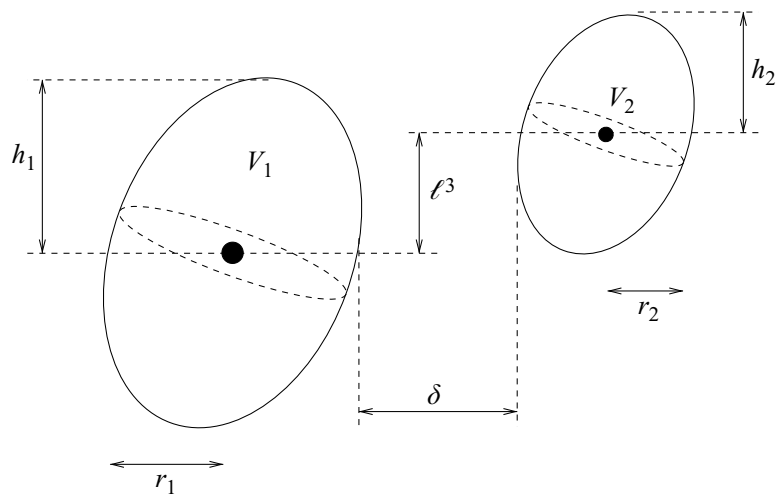

FIGURE 1. Definition of the geometrical parameters describing the initial conditions of a vortex interaction.

do not explicitly use the fact that the vortices have the same PV. In fact, the approach is general and the equations established to find equilibrium states and to address their linear stability are valid for any PV ratio. This is true even for opposite-signed vortices except in the particular situation where $\kappa_{1}=q_{1} V_{1} / 4 \pi=-q_{2} V_{2} / 4 \pi=-\kappa_{2}$. In this situation, the global centroid of the system (i.e. the centre of rotation) goes to $\infty$. The two vortices translate rather than rotate. Then, following the same logic, one has to determine the relevant translating reference frame rather than the relevant rotating frame.

\section{Numerical results}

\subsection{Comparison between the ELM and Contour Dynamics}

We measure the accuracy of the ellipsoidal approach by comparing the margin of stability obtained by the ELM with that obtained using the full QG equations. For the full equations, we use the Contour Dynamics (CD) method which represents vortices by a set of horizontal contours which are explicitly advected. The velocity field itself is obtained from the Biot-Savart law re-expressed in terms of contour integrals. The dynamical method is described in Dritschel (2002) while the algorithms used to find steady states together with their linear stability are described in Reinaud \& Dritschel (2002).

We first illustrate the results by describing one particular family of steady states. It corresponds to a couple of vortices with a unit volume ratio $\rho_{V}=V_{1} / V_{2}=1$, unit aspect ratios $h_{1} / r_{1}=h_{2} / r_{2}=1$, and no vertical offset $\ell^{3}=0$. As in Reinaud \& Dritschel (2002), the PV of each vortex is set to $2 \pi$ and the total volume of PV to $4 \pi / 3$. The general geometry of a vortex interaction is illustrated in figure 1.

For this family, we plot the angular impulse $J$, the energy $E$ and the growth rates of the modes $\sigma_{r}$ and their frequency $\sigma_{i}$ versus the horizontal gap $\delta$ in figure 2. The ELM results are in good agreement with the results from CD obtained in Reinaud \& Dritschel (2002). First, the angular impulse decreases as the gap decreases before reaching a minimum $J=J_{m}$ at a horizontal gap $\delta=\delta_{m}$ and then increases again. On the other hand, the energy exhibits the opposite trend but reaches its maximum $E_{M}$ at the same gap $\delta_{m}$. In addition, however, according to the linear stability analysis results, in figure 2(c), $\delta_{m}$ corresponds to the margin of stability. For $\delta>\delta_{m}$ all modes are neutrally stable $\left(\sigma_{r}=0\right)$, and all but one propagates (i.e. $\sigma_{i} \neq 0$ ). The modes 

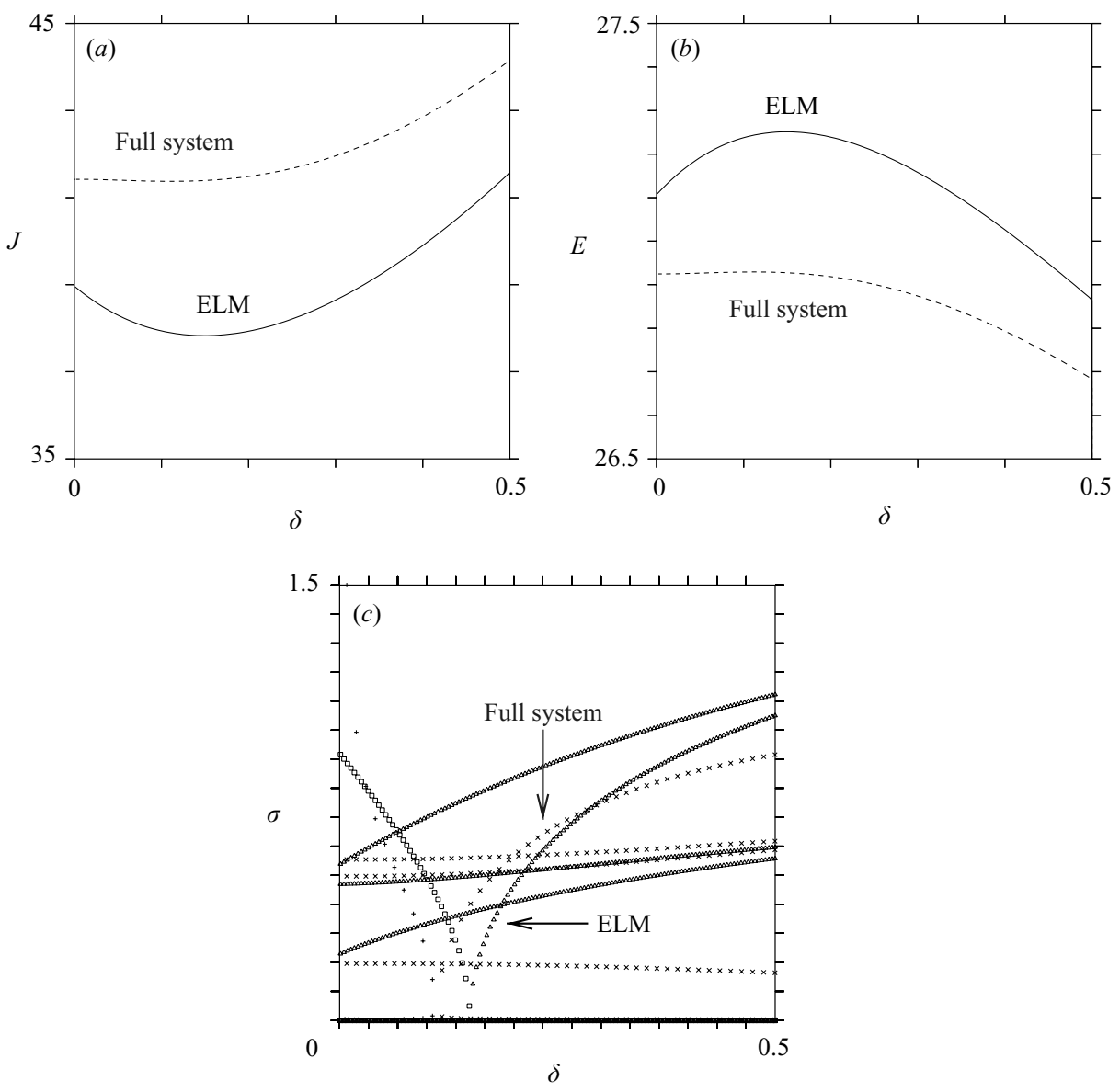

Figure 2. (a) Angular impulse $J,(b)$ energy $E,(c)$ frequencies $\sigma_{i}$ (triangles for ELM, crosses for $\mathrm{CD}$ ) and growth rates $\sigma_{r}$ (squares for ELM, pluses for CD) for two unit height-to-width aspect ratio, unit-volume-ratio, horizontally aligned vortices versus the horizontal gap $\delta$. In $(a)$ and (b) the solid line corresponds to the ELM results while the dashed line corresponds to the CD results (adapted from Reinaud \& Dritschel 2002).

are organized in pairs (if $\sigma=\sigma_{r}+\mathrm{i} \sigma_{i}$ is an eigenvalue, its conjugate $\sigma=\sigma_{r}-\mathrm{i} \sigma_{i}$ is also one). There are nine modes available from the ELM eigenvalue problem. Consequently one has a zero eigenvalue. This particular neutral mode corresponds to a global rotation of the system.

We see from figure 2(c) that one of the propagating modes (for $\delta>\delta_{m}$ ) has a frequency that collapses to zero more rapidly than the others. On reaching $\delta=\delta_{m}$ its frequency becomes zero (and remains zero for $\delta<\delta_{m}$ ) while its growth rate increases. Note that again these modes are organized in pairs and an unstable mode $\sigma=\sigma_{r}>0$ means that there exists a stable mode with $\sigma=-\sigma_{r}<0$. There is no surprise in these observations. They are consistent with what has been obtained in Reinaud \& Dritschel (2002) when solving the full equations - see also Dritschel (1995) and Meunier et al. (2002) for analogous results in two-dimensional flows. Besides, the values of the critical angular impulse are in good agreement, with $J_{m}(\mathrm{ELM})=37.829$ while $J_{m}(\mathrm{CD})=41.366$, a difference of $8.55 \%$, while for the energy we have $E_{M}(\mathrm{ELM})=27.251$ and $E_{M}(\mathrm{CD})=26.928$, a difference of $1.20 \%$. The critical 


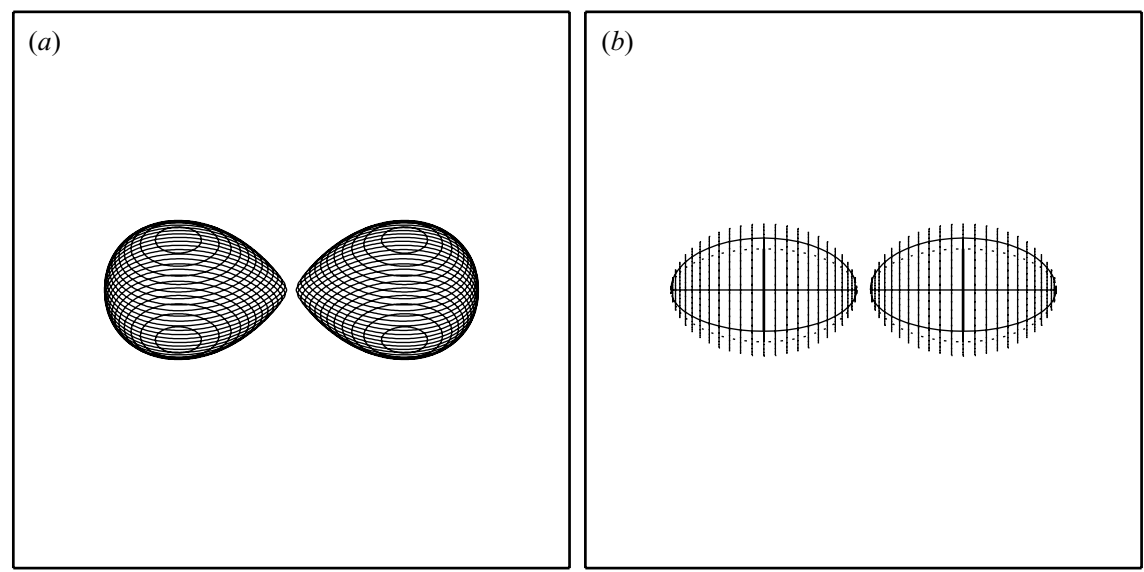

FIGURE 3. Shape of the critical steady states for two unit-aspect-ratio, unit-volume-ratio, horizontally aligned vortices: $(a)$ the exact shape; $(b)$ the ellipsoidal approximation. In this and subsequent figures, the vortices are viewed orthographically at an angle of $60^{\circ}$ from the vertical.

horizontal offset $\ell_{c}^{1}$ agrees within $5.86 \%$. It is therefore arguable that the merger-type instability observed previously in the full equations is consistent with a pure ellipsoidal mode, and hence the simplified approach filtering non-ellipsoidal perturbations is able to give an approximate yet relevant estimate of the critical merger distance in this situation. The actual shape of the vortices at the margin of stability is shown in figure 3 . It is seen that the exact vortices are asymmetric with sharp innermost edges and smooth outermost edges. The non-ellipsoidal asymmetry is responsible for the inexact match between the two approaches.

We continue the comparison by examining the other cases investigated in Reinaud \& Dritschel (2002) having a vertical offset between the vortices (for otherwise identical parameters, $\left.\rho_{V}=1, h_{1} / r_{1}=h_{2} / r_{2}=1\right)$. Figure 4 presents the dependence of the critical angular impulse $J_{m}$, the critical energy $E_{M}$, the critical horizontal offset $\ell_{c}^{1}$ and the critical total distance $d_{c}$ on the relative vertical offset $\ell^{3} / r$, all at the margin of stability. Overall, there is a good agreement ( $1 \%$ to $10 \%$ differences). It is seen that global tendencies are respected although as far as $\ell_{c}^{1}$ and $J_{m}$ are concerned, the ELM results show a small increase of the values for intermediate vertical offsets. Recall that $J$ is strongly related to $\ell^{1}$. Considering the evolution of the errors, we may say that $J_{m}$ and $\ell^{1}$ are in fact less consistent for small vertical offsets. In terms of accuracy, the results are summarized in figure 5 where the relative differences at the margin of stability for $J_{m}, E_{M}, \ell_{c}^{1}$ and $d_{c}$ are plotted versus the relative vertical offset $\ell^{3} / r$ where $r$ is the mean horizontal radius common to both vortices. This shows that the ELM is able to predict $J_{m}$ to within $8 \%, \ell_{c}^{1}$ to within $5 \%$, and $E_{M}$ to within $1 \%$. As noted above, the errors are larger for small vertical offsets. We attribute this to the fact that the full steady states are less ellipsoidal in these cases. Indeed, it can be seen from the results in Reinaud \& Dritschel (2002), in particular in their figure 2, that the innermost edges are sharper when vortices are horizontally aligned.

\subsection{The margin of stability for oblate to moderately prolate vortices}

We next present a summary of the ELM results obtained for oblate to moderately prolate vortices, namely $h / r \leqslant 1.6$. Vortices taller than this are seldom found in turbulence (Reinaud et al. 2003). We consider 15 values for the aspect ratio of each 

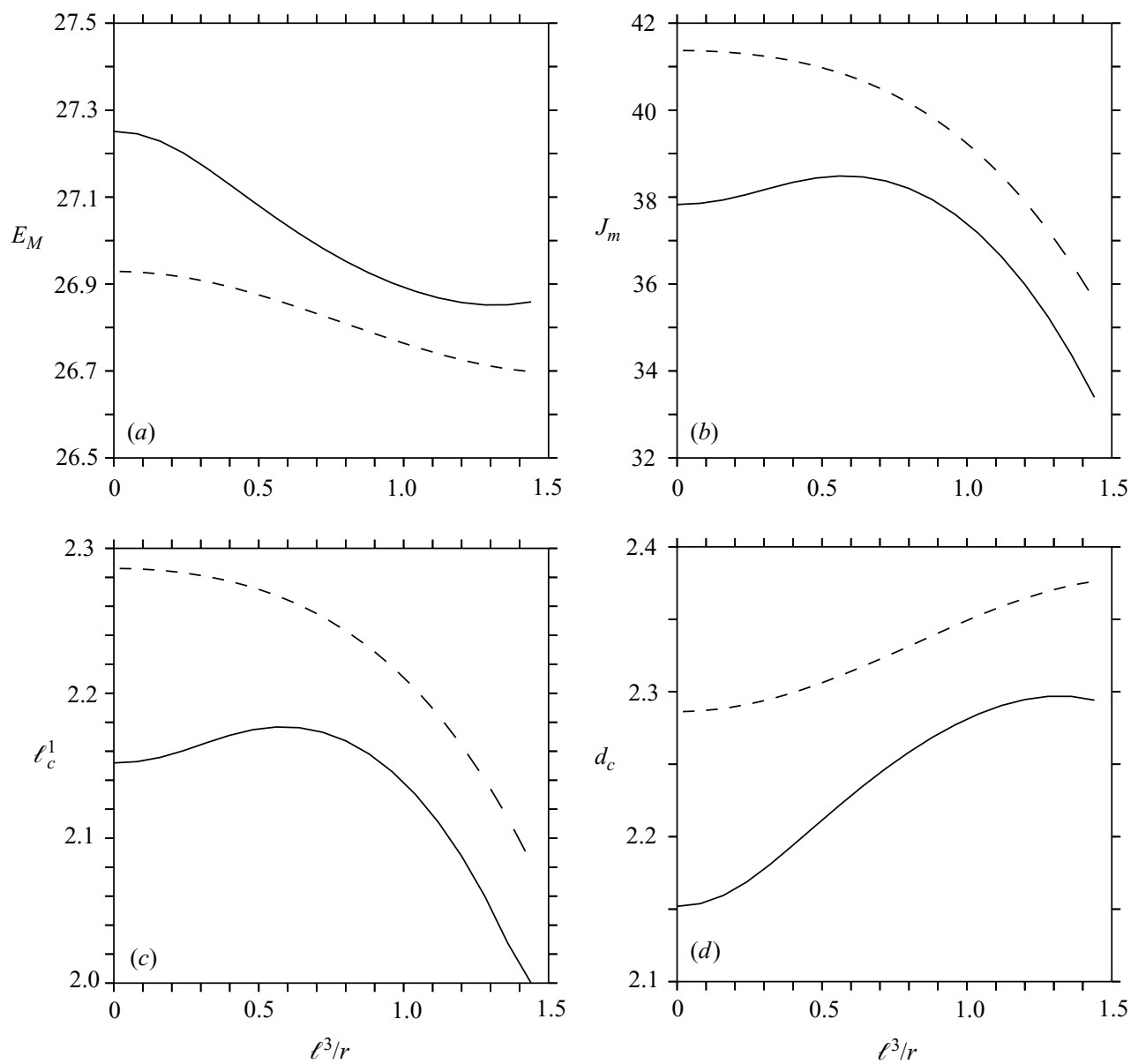

FiguRE 4. (a) Critical energy $E_{M},(b)$ critical angular impulse $J_{m},(c)$ critical horizontal distance $\ell_{c}^{1}$ and $(d)$ the total critical centroid separation $d_{c}$, versus the relative vertical offset $\ell^{3} / r$ for two equal-volume, unit-aspect-ratio vortices. The dashed lines corresponds to the results obtained in Reinaud \& Dritschel (2002) using the full QG equations, and the solid lines correspond to the ELM results.

vortex, equally spaced between $0.2 \leqslant h / r \leqslant 1.6$. This is done for various volume ratios between the two vortices $\rho_{V}=V_{1} / V_{2}$ as well as for various vertical offsets $\ell^{3}$. Specifically, we consider five volume ratios equally spaced in the range $[0.2,1]$. The vertical offset is taken as a fraction of $h_{1}+h_{2}$. This distance corresponds to the limit above which the two vortices no longer share any common horizontal level. We consider five different vertical offsets from $0.005\left(h_{1}+h_{2}\right)$ to $0.8\left(h_{1}+h_{2}\right)$. More precisely we use $0.005,0.2,0.4,0.6$ and 0.8 as fractions for the vertical offset. For the nearly aligned case $\ell^{3}=0.005\left(h_{1}+h_{2}\right)$, we also slightly offset the volume ratios by a factor $0.99^{3}$, namely the volume ratios are $\rho_{V} \simeq 0.1941,0.3881,0.5822,0.7762$, and 0.9703 , in these cases. This particular setup prevents perfectly symmetric equilibria and is justified in the next subsection.

We seek to determine the margin of stability in the resulting 5625 situations. As described in $\S 2$, for given values of the aspect ratios, volume ratio and vertical offset, we start the investigation with two well-separated spheroids. The initial horizontal 


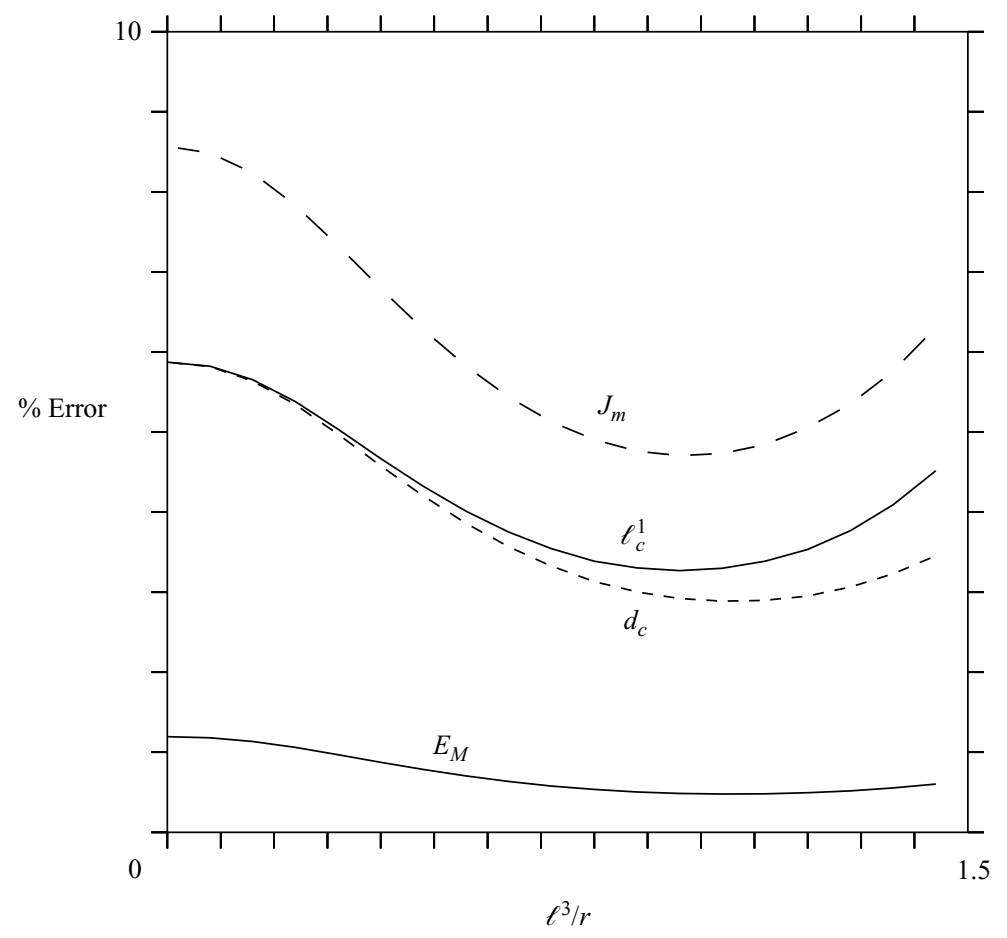

FIGURE 5. Percentage difference for the critical energy $E_{M}$, angular impulse $J_{m}$, horizontal distance $\ell_{c}^{1}$, and total centroid distance $d_{c}$ between the exact equilibrium vortices and the approximate ones obtained by the ELM versus the relative vertical offset $\ell^{3} / r$. The vortices have the same volume $\left(\rho_{V}=1\right)$ and have unit aspect ratios $\left(h_{1} / r_{1}=h_{2} / r_{2}=1\right)$.

centroid separation $\ell$ is set to $2\left(r_{1}+r_{2}\right)$ where $r_{1}$ and $r_{2}$ are respectively the horizontal radii of the vortices. When a steady state is obtained the horizontal gap $\delta$ is reduced by $2 \times 10^{-4}$. The procedure is continued until the energy of the state is seen to decrease (indicating that we have reached the margin of stability assuming the criterion previously proposed is general). We then perform a linear stability analysis for four neighbouring states surrounding the observed maximum of energy. A match between the location $\delta_{m}$ of the maximum of energy (which always coincides with a minimum in the angular impulse) and the margin of stability is obtained if the first state, with $\delta>\delta_{m}$, is neutrally stable and the last of the four states, with $\delta<\delta_{m}$, is linearly unstable. Such a match is observed in all of our converged results.

We present next contour plots summarizing several key properties of the equilibrium states at the margin of stability. The contours are plotted in the plane $\left(h_{1} / r_{1}, h_{2} / r_{2}\right)$ for all values of the vertical offset $\ell^{3}$ and of the volume ratio $\rho_{V}$ considered. Recall that vortex 2 is the larger one. The raw data consist then of 225 discrete values in the $h_{1} / r_{1}, h_{2} / r_{2}$ plane for each value of $\ell^{3}$ and $\rho_{V}$.

Despite many attempts, the margin of stability could not be reached in a few cases. These critical cases are encountered in a specific region of parameter space where the vertical offset is small, and when at least one of the vortices has an aspect ratio close to but greater than unity. In these cases, the algorithm used to reach equilibrium states becomes unstable and oscillates when one of the vortices exhibits a nearly circular cross-section in the $(x, z)$-plane. These difficulties appear to be associated with a spurious oscillating numerical mode arising from the discrete 


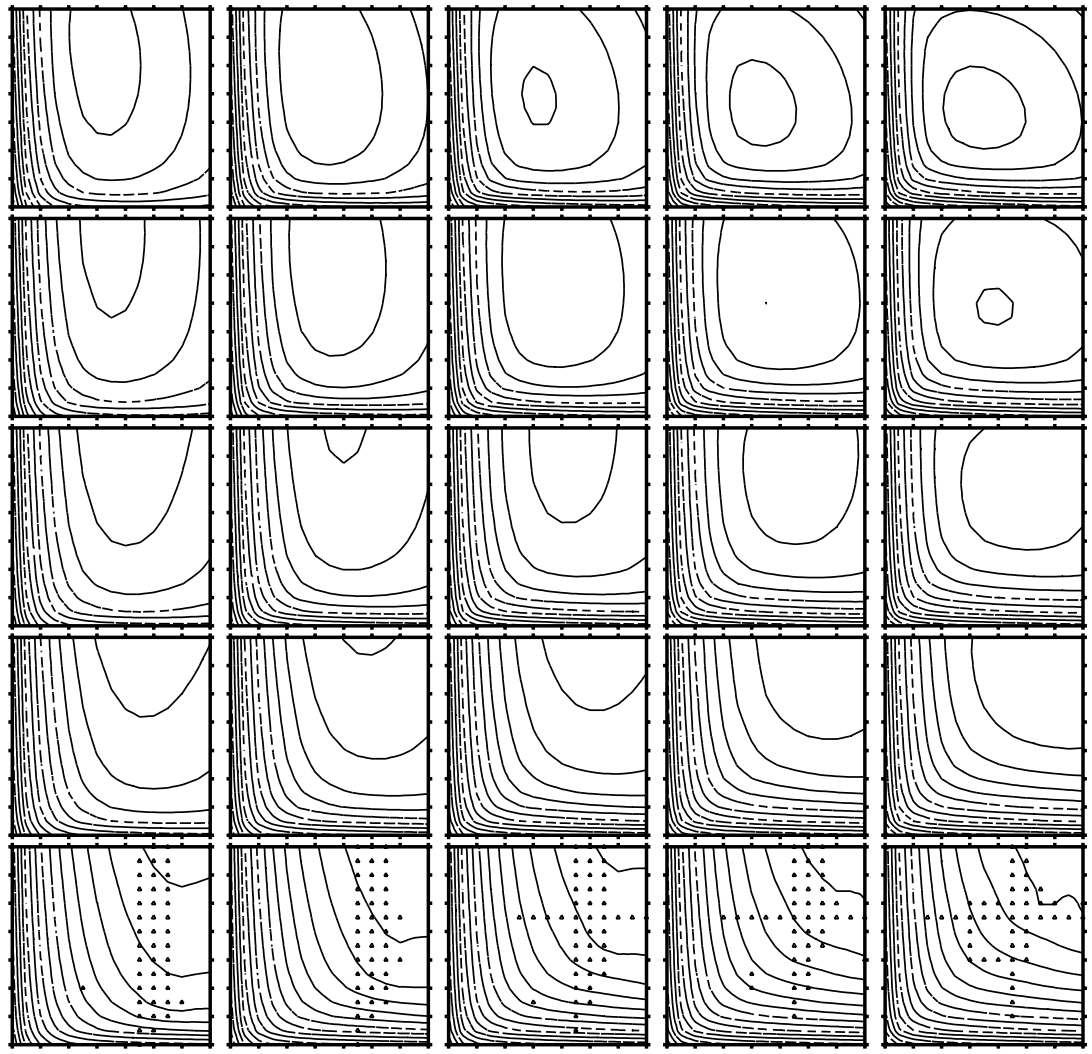

FIGURE 6. Iso-levels of centroid separation $d_{c}$ between the two vortices at the margin of stability. The $x$-axis of each graph corresponds to the aspect ratio of the smaller vortex for $0.2<h_{1} / r_{1}<1.6$, while the $y$-axis corresponds to that of the larger vortex for $0.2<h_{2} / r_{2}<1.6$. Graphs along the same row correspond to cases with a fixed vertical offset, with $\ell^{3}=0.8\left(h_{1}+h_{2}\right)$ at the top and $\ell^{3}=0.005\left(h_{1}+h_{2}\right)$ at the bottom. The vertical offset differs by $0.2\left(h_{1}+h_{2}\right)$ for two adjacent rows, except for the last two rows, see text for details. Each column of graphs corresponds to a fixed volume ratio, with $\rho_{V}=0.2$ at the left and $\rho_{V}=1$ at the right. The volume ratio differs by 0.2 between two adjacent columns. The first solid contour is for $d_{c}=2.5$, other solid contours correspond to smaller values while the dashed contours correspond to larger values. The contour interval is 0.1 . The very small symbols $\Delta$ mark points where data are interpolated only.

approximation used to model the external flow induced by the ellipsoids. Increasing the number of singularities from 4 to 7 to 13 reduces the extent of the spurious region. However, this numerical mode only affects a small region of parameter space where one of the vortices exhibits a nearly circular cross-section and the vortices are horizontally aligned. In these parts of the parameter space, we interpolate the data from nearby converged states to draw the contour plots. We use a procedure based on cubic interpolation. The interpolation is done in the $h_{1} / r_{1}, h_{2} / r_{2}$ plane and is one-dimensional, i.e. we interpolate 'horizontally' (from points with $h_{2} / r_{2}$ fixed) or 'vertically' (from points with $h_{1} / r_{1}$ fixed). The interpolated data are indicated in the contour plots by very small triangles.

We first examine the critical centroid separation $d_{c}$ between the two vortices, see figure 6 . We indicate a few general trends that describe the influence of the parameters $\left(h_{1} / r_{1}, h_{2} / r_{2}, \ell^{3}\right.$ and $\left.\rho_{V}\right)$ on the critical merger distance. It is seen first that when 
the smaller vortex is flat $\left(h_{1} / r_{1} \ll 1\right), d_{c}$ depends only weakly on the aspect ratio of the larger vortex $h_{2} / r_{2}$. A similar influence is observed if $h_{2} / r_{2} \ll 1$ but only if the small vortex is not flat. This trend can be explained in the following way. Our results indicate that, for a given volume ratio and vertical offset, the shape of the flat vortex and the strain induced on it (at the margin of stability) depends only weakly on $h / r$ of the other vortex. Moreover, the eigenvector of the unstable mode at the margin of stability indicates that this mode acts predominantly on the centroid separation $\ell^{2}$ and on the deformation of the flat vortex. These observations indicate that $d_{c}$ is controlled by the flat vortex.

We also observe (at least in the range of aspect ratios considered) that if the vertical offset is small, $\ell^{3}<0.4\left(h_{1}+h_{2}\right), d_{c}$ decreases monotonically with both aspect ratios. For larger vertical offsets, $d_{c}$ may reach a local minimum. For the largest vertical offsets investigated and for a moderate volume ratio, we may roughly say that the extremum is observed when the two vortices have comparable aspect ratios, around 1. This appears to be a consequence of the ability of vortices with $h / r \neq 1$ to distort and tilt more easily than spherical vortices. These deformations help bring the innermost edges of the vortices close enough together to induce instability.

Regarding the influence of the volume ratio on $d_{c}$, we see that the graphs for $\rho_{V} \neq 1$ are strongly asymmetric with respect to the symmetry line $h_{1} / r_{1}=h_{2} / r_{2}$. The general trend is that the critical distance between a large vortex with $h_{2} / r_{2}=a$ and a smaller one with $h_{1} / r_{1}=b<a$ is larger than that in the complementary situation consisting of the interaction between a large vortex with $h_{2} / r_{2}=b$ and a smaller one with $h_{1} / r_{1}=a$. Again, one may put forward geometrical arguments to explain this trend. A tall vortex can be tilted easily and this allows the centroid of the vortex to be displaced further away while the innermost edges of the vortices remain close to one another. Finally, a moderate vertical offset usually increases the critical merger distance, as was observed in Reinaud \& Dritschel (2002).

Meunier et al. (2002), in their study of two-dimensional merger, proposed the idea that the critical merger distance $d_{c}$ is approximately proportional to a length scale derived from the angular impulse, namely $l_{J}=\sqrt{J / \Gamma}$ where $\Gamma$ is the volume integral of PV. We have tested this idea in the three-dimensional quasi-geostrophic context and have not found a significant correlation between $d_{c}$ and $l_{J}$ over the full parameter space.

Contour plots for the critical $E_{M}$ energy are given in figure 7. Energy decreases when the volume ratio increases toward 1 . The influence of the vertical offset is relatively small and tends to weaken the interaction (reduce energy). Also, when the volume ratio is small, i.e. vortex 2 is much larger than vortex 1 , and when vortex 2 is a lens-like vortex $\left(h_{2} / r_{2} \ll 1\right)$, the energy is almost independent of the aspect ratio of the first vortex, and of the vertical offset. In this situation the energy of the flow is principally contained within vortex 2 which feels only weakly the influence of vortex 1 . On the contrary, when vortex 2 is less oblate, the flow is more energetic and more sensitive to the aspect ratio of the smaller vortex 1 . In these situations, the vortices are closer to each other and the interaction energy is important. We show this by plotting separately the self-energy of both vortices $E_{1}$ and $E_{2}$, and the interaction energy $E_{b}$ in figure 8 for the case $\rho_{V}=0.2$ and $\ell^{3}=0.2\left(h_{1}+h_{2}\right)$. The energy of vortex 2 is roughly an order of magnitude larger than both the energy of vortex 1 and the interaction energy. The combined influence of vortex 1 and the interaction energy explains the dependence of the total energy on $h_{1} / r_{1}$. For $h_{2} / r_{2} \ll 1$, both $E_{1}$ and $E_{b}$ depend more weakly on $h_{1} / r_{1}$; hence, the total energy is virtually independent of $h_{1} / r_{1}$. Note that taller vortices are more energetic because they rotate faster. 


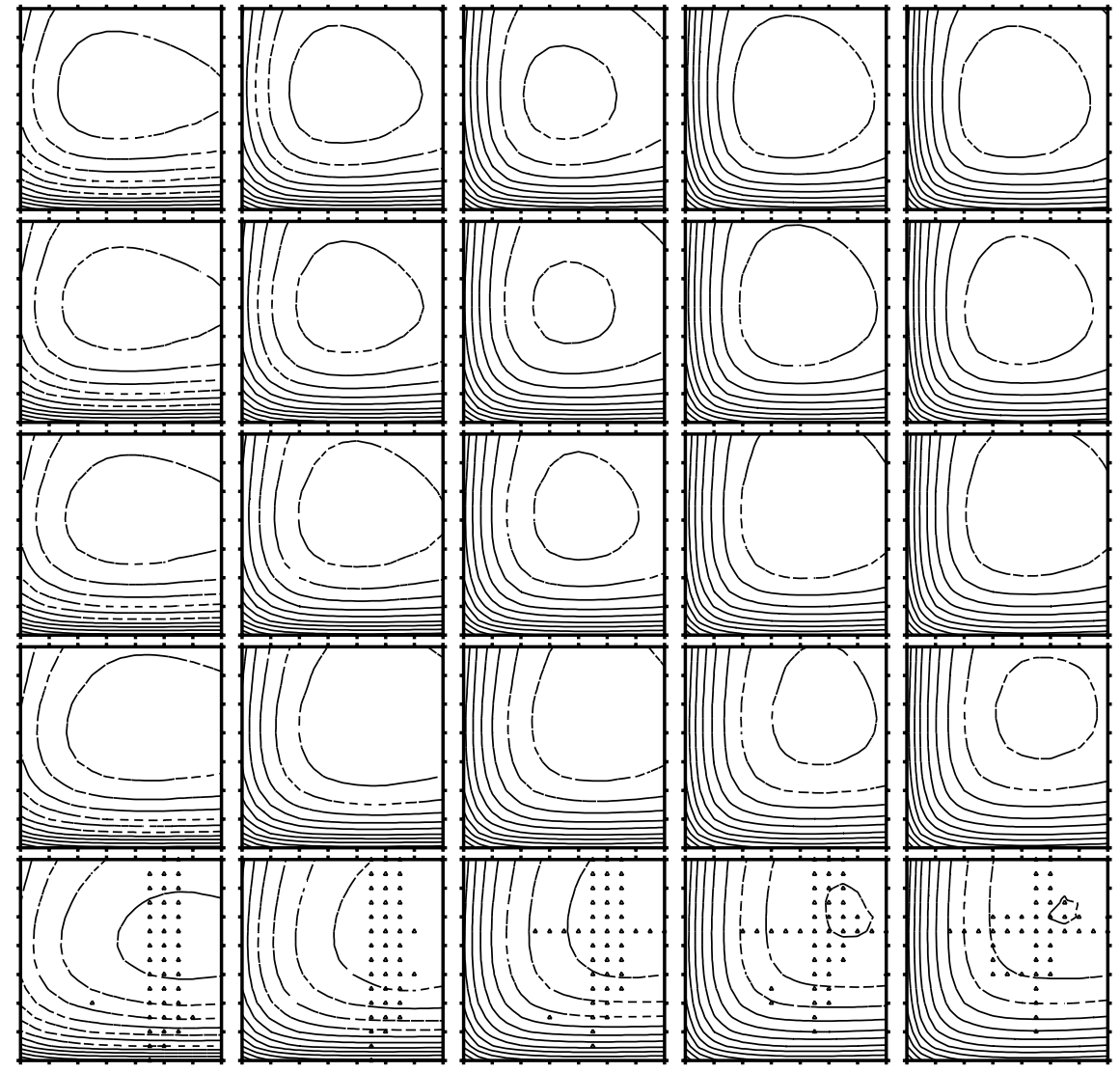

FiguRE 7. Iso-levels of the energy $E_{M}$ at the margin of stability. The layout is the same as in figure 6. The first solid contour is for $E_{M}=26$, and the contour interval is 0.5 .

(a)

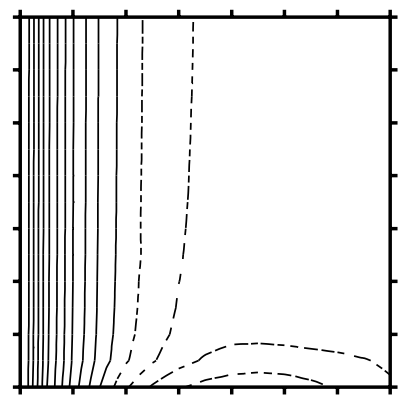

(b)

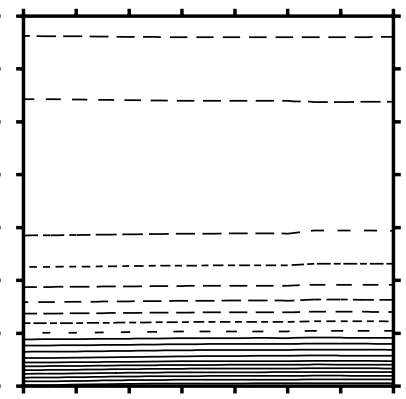

(c)

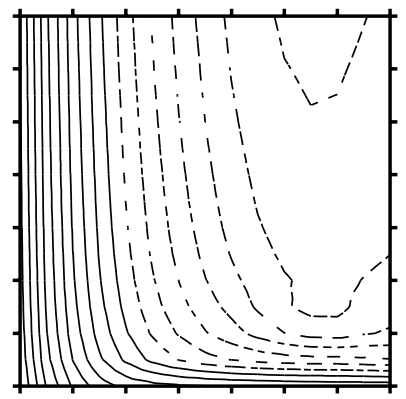

FIGURE 8. Contour plots for the components of the energy at the margin of stability in the case where $\rho_{V}=0.2$ and $\ell^{3}=0.2\left(h_{1}+h_{2}\right)$. (a) Self-energy of vortex $1, E_{1}$. The first solid contour is for $E_{1}=1.5$, and the contour interval is 0.02 . (b) Self-energy of vortex $2, E_{2}$. The first solid contour is for $E_{2}=22.5$, and the contour interval is 0.25 . (c) Interaction energy $E_{b}$. The first solid contour is for $E_{b}=3$, and the contour interval is 0.08 .

This subsection has described in broad terms the margin of stability for interacting QG vortices. But a major question remains to be answered. It concerns perfectly horizontally aligned vortices - situations which have been the primary focus of past 


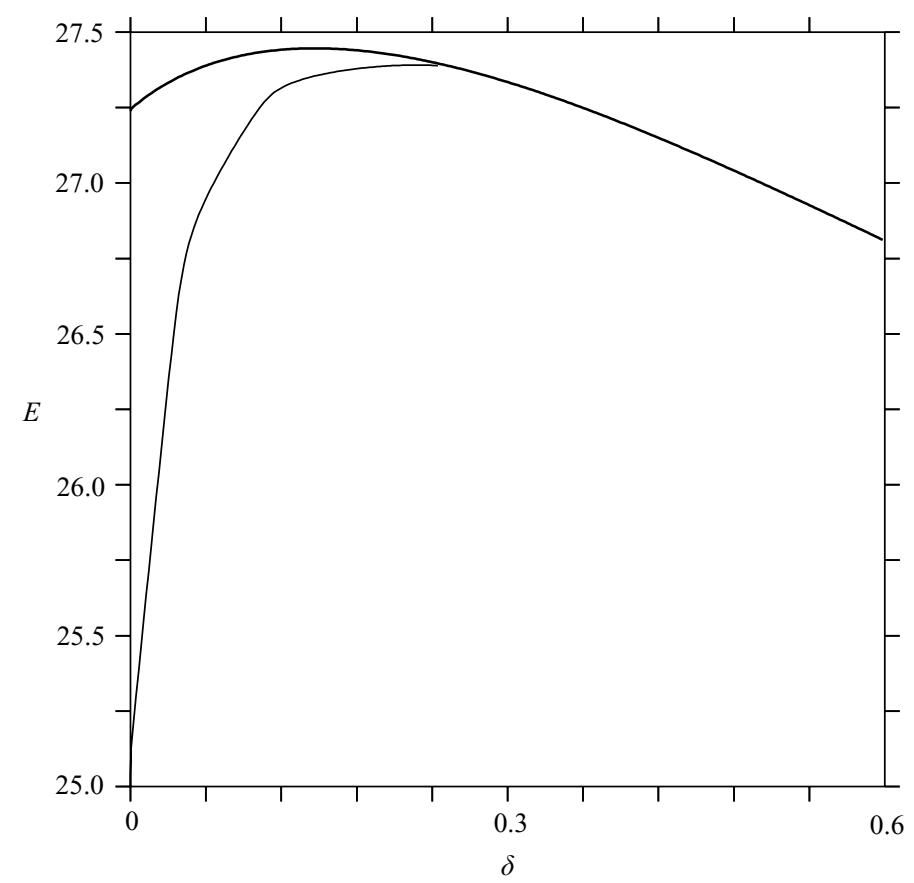

FigURE 9. Energy $E$ as a function of the gap $\delta$ for $\rho_{V}=1.0, h_{1} / r_{1}=1.5, h_{2} / r_{2}=1.1$, and $\ell^{3}=0$. The bold line indicates the branch of symmetric vortices, the thin line to the left indicates the branch of asymmetric vortices.

reaearch - which, for good reasons, we avoided in this subsection, but which we will discuss next.

\subsection{The tilt instability}

We now focus on horizontally aligned vortices, $\ell^{3}=0$. In these cases, without any seed for asymmetry, the equilibrium vortices remain perfectly symmetric with respect to the $(x, y)$-plane. Von Hardenberg et al. (2000) and Dritschel (2002) focused on such perfectly symmetric initial conditions. Since the equations preserve symmetry, only symmetric perturbations were observed. In these cases, asymmetry can only arise from the low numerical noise of the solution methods, arguably too small to induce any noticeable asymmetric behaviour over a moderate integration time. Illustrations of the resulting symmetric nonlinear interactions are provided in both papers. We argue that the imposed symmetry can be misleading. Our present stability results indicate that prolate vortices $(h / r>1)$ are in fact sensitive to asymmetric modes and, more than this, these unstable modes occur at greater separation distances than do the symmetric instabilities.

We consider a first example of two vortices with a volume ratio of unity and aspect ratios of $h_{1} / r_{1}=1.5$ and $h_{2} / r_{2}=1.1$ respectively. We compute the steady states for this family, as done in the previous subsection except for a slight difference. Here, the vertical offset is exactly zero and we enforce explicitly that the vortices are symmetric with respect to the $(x, y)-,(x, z)-$, and $(y, z)$-planes. This is done straightforwardly by imposing that the two shape matrices remain diagonal. The evolution of the total energy as a functionof the gap $\delta$ is shown as the bold curve in figure 9. Again, 


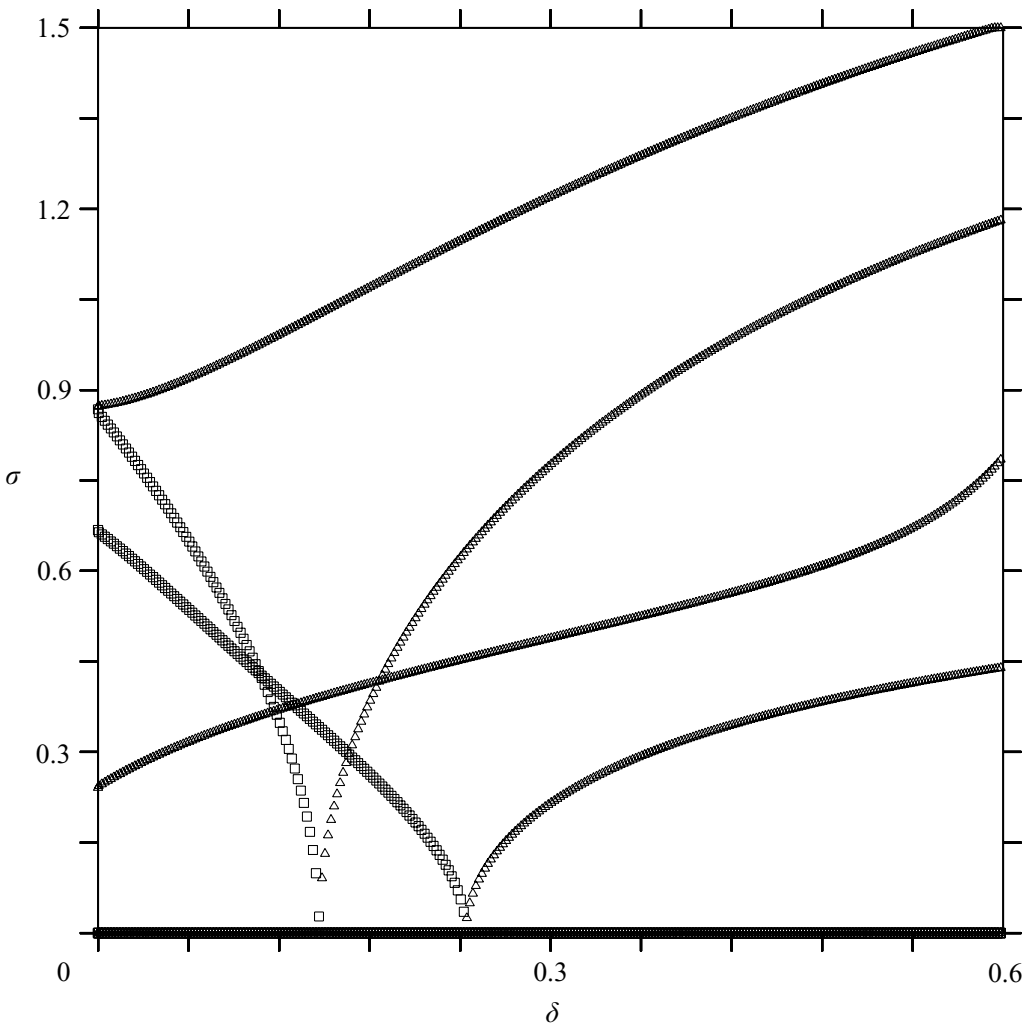

FIGURE 10. Growth rate $\sigma_{r}$ (squares) and frequencies $\sigma_{i}$ (triangles) of the modes as a function of the gap $\delta$ for $\rho_{V}=1.0, h_{1} / r_{1}=1.5, h_{2} / r_{2}=1.1$, and $\ell^{3}=0$.

the energy reaches a maximum as a function of the gap. The growth rates of the instability modes are presented in figure 10. It is seen that the maximum of energy, $E_{M}=27.44649$ at $\delta=0.146467$, corresponds to the emergence of an instability. But, clearly the situation differs from the ones discussed previously, since now the local extremum of energy does not correspond to the margin of stability - a mode of instability is encountered previously, i.e. at a greater gap. Note that the onsets of both instabilities are associated with a mode whose frequency collapses to zero.

We now aim to describe the instability occurring before the extremum of energy for the symmetric vortices. The instability occurs at $\delta=0.2424616$, well before the gap corresponding to the extremum of energy. This instability is associated with a new bifurcation in the set of solutions. There is in fact another branch of solutions arising from this point corresponding to unstable asymmetric solutions. We have been able to follow this new branch by introducing a seed for asymmetry on the stable symmetric state just before the bifurcation - slightly offsetting the centroids in the vertical then resuming the algorithm without imposing symmetry. By taking smaller and smaller vertical offsets, we have verified that this asymmetric branch coincides with the onset of instability on the symmetric branch. The asymmetric branch obtained is also unstable. Moreover, the energy of the asymmetric solutions decreases with $\delta$ after this bifurcation, see figure 9. This indicates that, in fact, the margin of stability is associated with an extrema of energy as thought. The symmetric branch of solutions is just not the relevant one to follow in this case. 


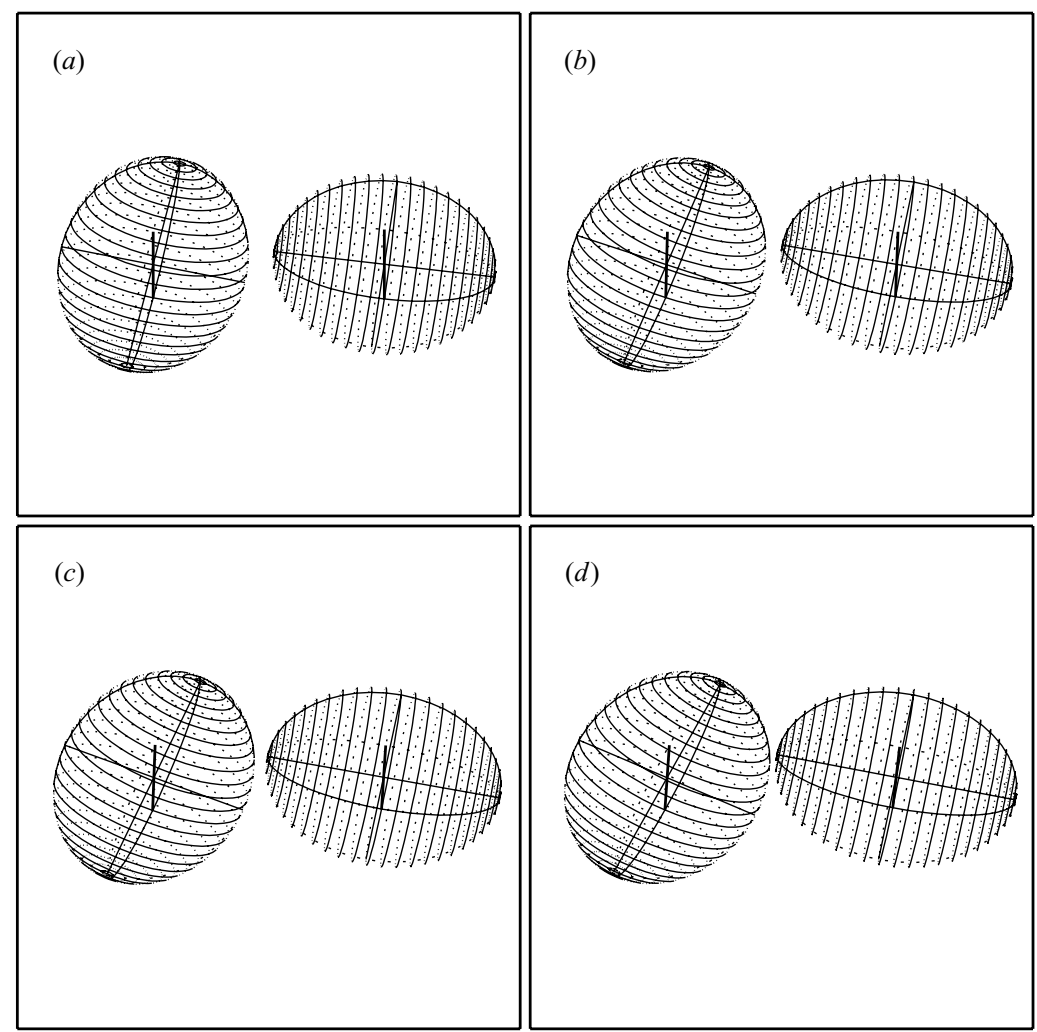

FIGURE 11. Asymmetric unstable state (case A): the vortices have mean aspect ratios of 1.5 and 1.1. The volume ratio $\rho_{V}=1$ and the vertical offset $\ell^{3}=0.004\left(h_{1}+h_{2}\right)$. Times shown are (a) $t=0,(b) 351,(c) 353,(d) 353$. The initial gap is $\delta=0.214463$.

We next illustrate the instability observed on both branches. We first consider an asymmetric case, with a gap $\delta=0.214463$, hereinafter referred to as case A. The growth rate of the unstable mode is $\sigma_{r}=0.106503$. The vortex interaction is shown in figure 11. The equilibrium consists of two vortices tilted toward each other. The instability itself causes the vortices to tilt further toward each other and eventually touch (an indication of eventual merger, based on comparisons with CD). We show the symmetric case for the same gap $\delta=0.214463$ in figure 12 , hereinafter referred to as case B. The growth rate of the unstable mode is higher here than in the previous case with $\sigma_{r}=0.170874$. A similar behaviour is observed. The vortices eventually tilt toward each other, breaking the symmetry of the initial conditions. The flow is sensitive to asymmetric perturbations. Note that the way in which the vortices merge is very similar in the two cases, apart from the orientation, randomly seeded in the symmetric case by numerical noise. In fact, surprisingly, the dynamical behaviour is nearly identical. We illustrate this by showing in figure 13 the absolute value of the tilt angle for vortex 1 in each case The tilt angle is define as the angle between the major axis of the vortex and the vertical. The two curves almost exactly superimpose except for a shift in time of $\Delta t=37.25$.

Note that, by symmetry, a third branch of asymmetric solutions must exist having the opposite tilt angle for both vortices. However, this different branch must have exactly the same properties $(E, J)$, in other words, the two asymmetric branches 


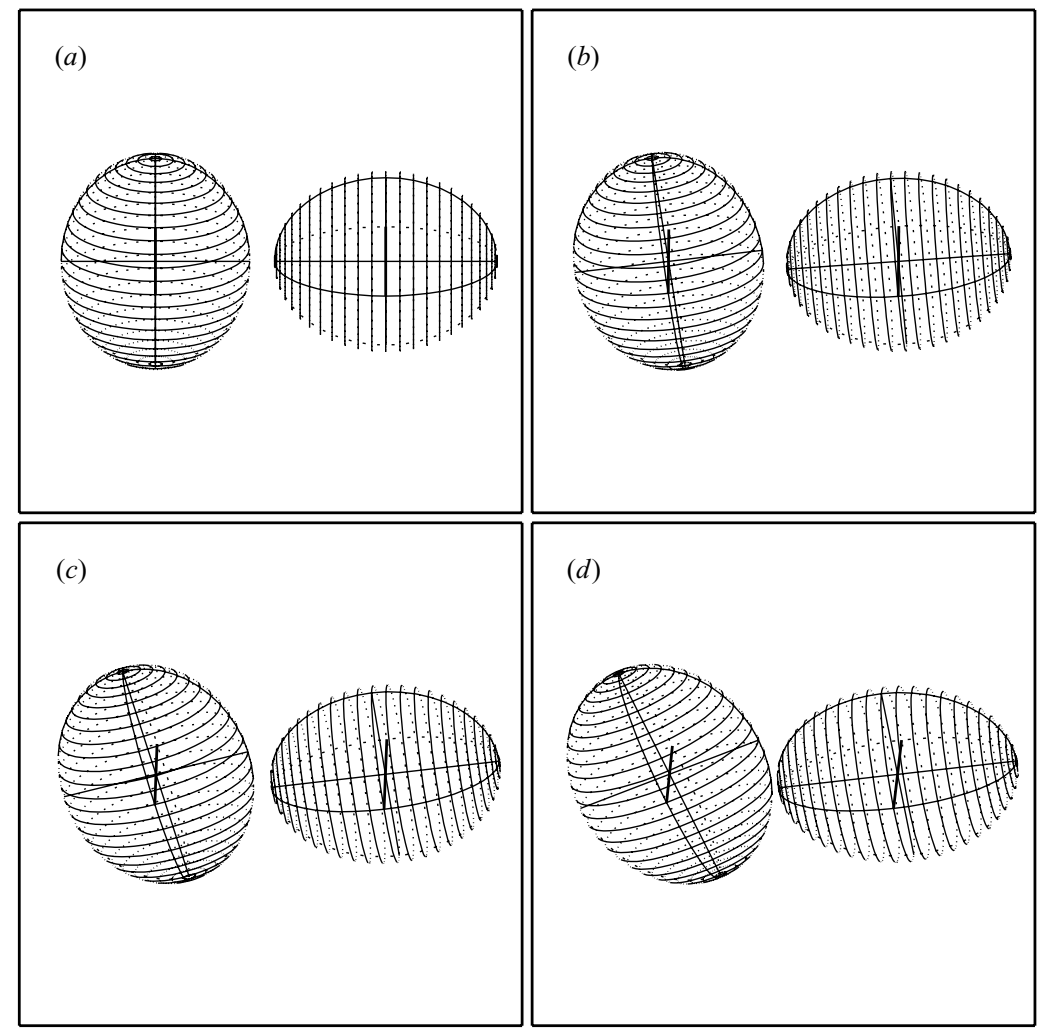

FigurE 12. Symmetric unstable state (case B): the vortices have mean aspect ratios of 1.5 and 1.1. The volume ratio $\rho_{V}=1$ and the vertical offset $\ell^{3}=0$. Times shown are $(a) t=0,(b) 310$, (c) $314,(d) 316$. The initial gap is $\delta=0.214463$.

are equivalent. The bifurcation is similar to a pitchfork bifurcation. Analogous bifurcations with symmetry breaking were observed by Saffman (1992) for twodimensional vortices.

As mentioned above, this instability is observed when at least one of the vortices is prolate. To more clearly illustrate this instability, we consider two taller, horizontally aligned, equal-volume vortices. The aspect ratio is set to 4 . Again, we compute the family of symmetric states and we identify the first unstable mode. Then we compute the asymmetric equilibrium branch arising from this bifurcation. The dependence of the energy $E$ on the gap $\delta$ is given in figure 14. Again, the symmetric branch of solutions exhibits a maximum of energy $E_{M}=27.45644$ at $\delta=\delta_{m}=0.162387$. Yet again, the equilibria are unstable before $\delta_{m}$. The first mode of instability is found at $\delta=1.12761$, and an unstable asymmetric branch of solutions bifurcates at this point. For this new branch, the energy decreases as the gap decreases, indicating that the margin of stability coincides with the maximum of energy (we can trace back the complete asymmetric branch for distant vortices and it is seen to follow the symmetric branch until it bifurcates at the margin of stability). We next illustrate unstable interactions on both branches of solutions. First, figure 15 shows one of the asymmetric branches. The initial gap is $\delta=1.01758$ and the growth rate of the unstable mode is $\sigma_{r}=0.100125$. As before the two vortices tilt toward each other before touching (which corresponds to merger in the full equations, as verified 


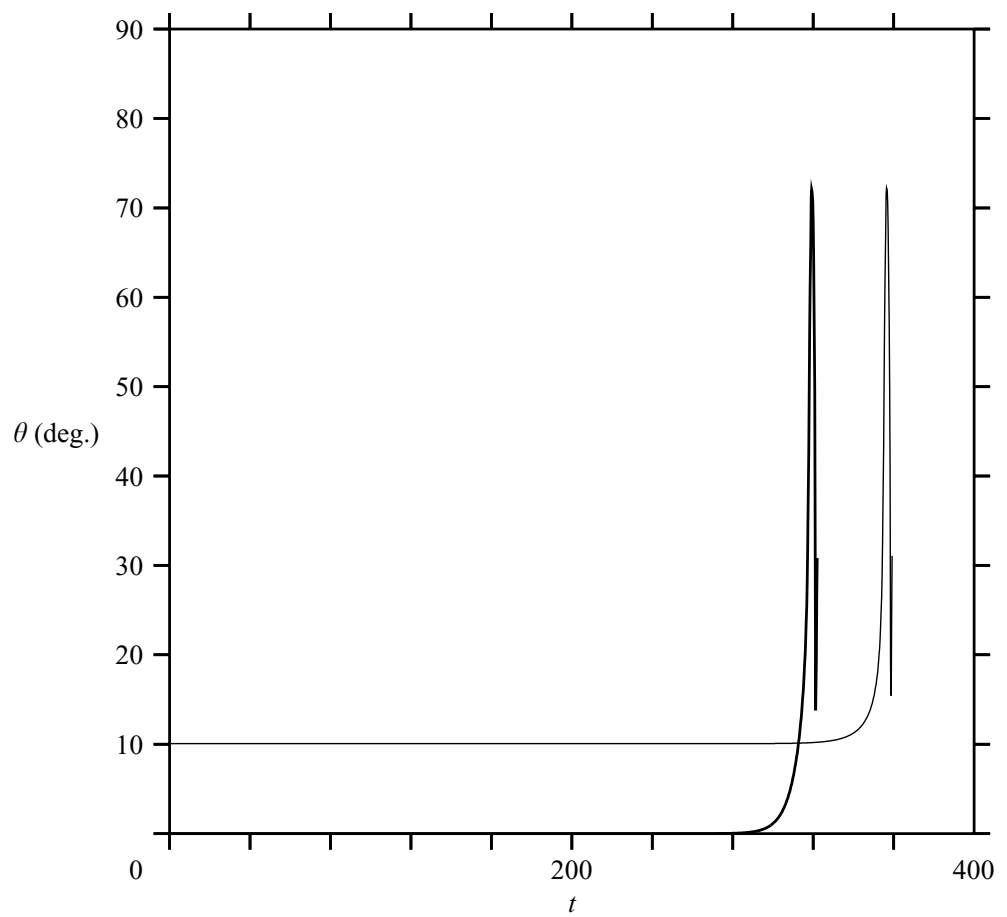

Figure 13. Evolution of the tilt angle for vortex 1: case A (thin line), case B (bold line).

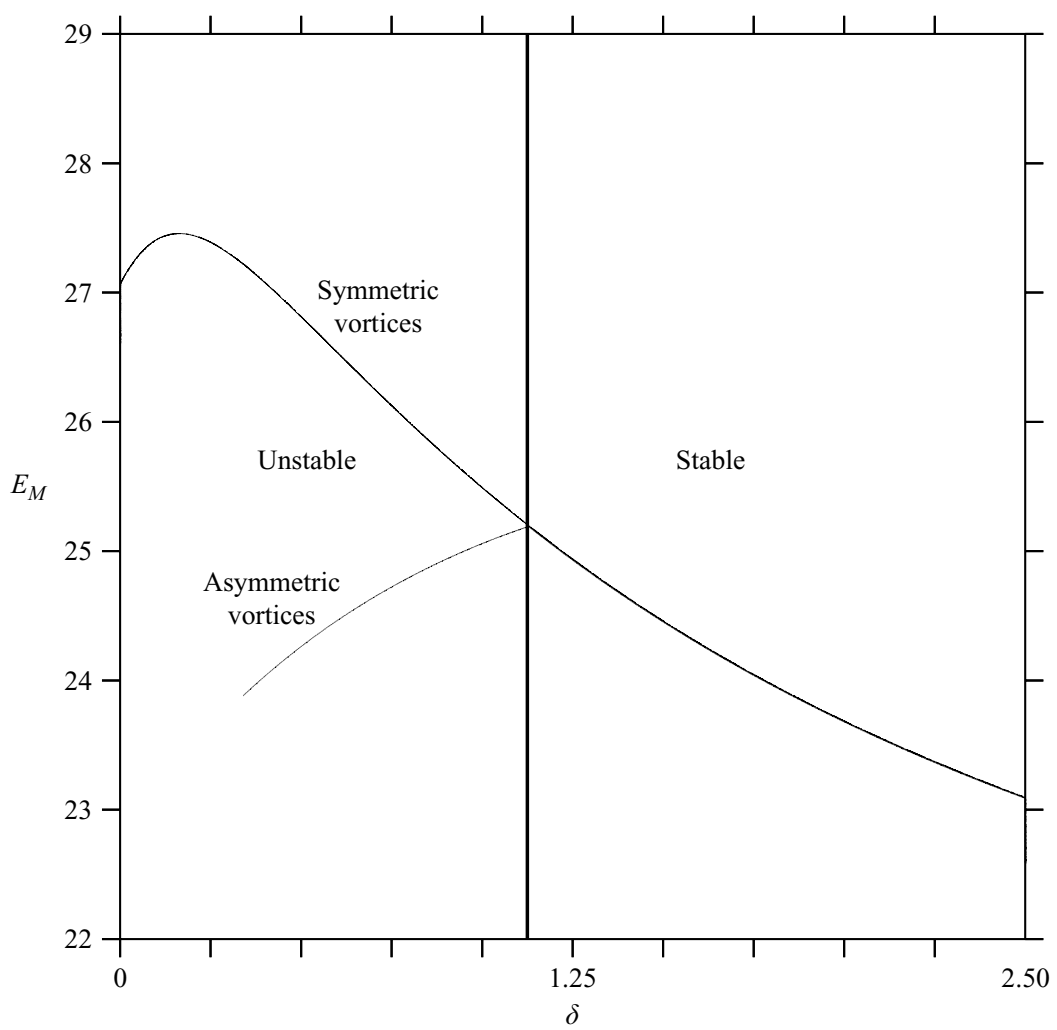

FIGURE 14. Energy $E$ as a function of the gap $\delta$ for $\rho_{V}=1.0, h_{1} / r_{1}=h_{2} / r_{2}=4$, and $\ell^{3}=0$. The vertical line separates the unstable region (left) and the stable region (right). 


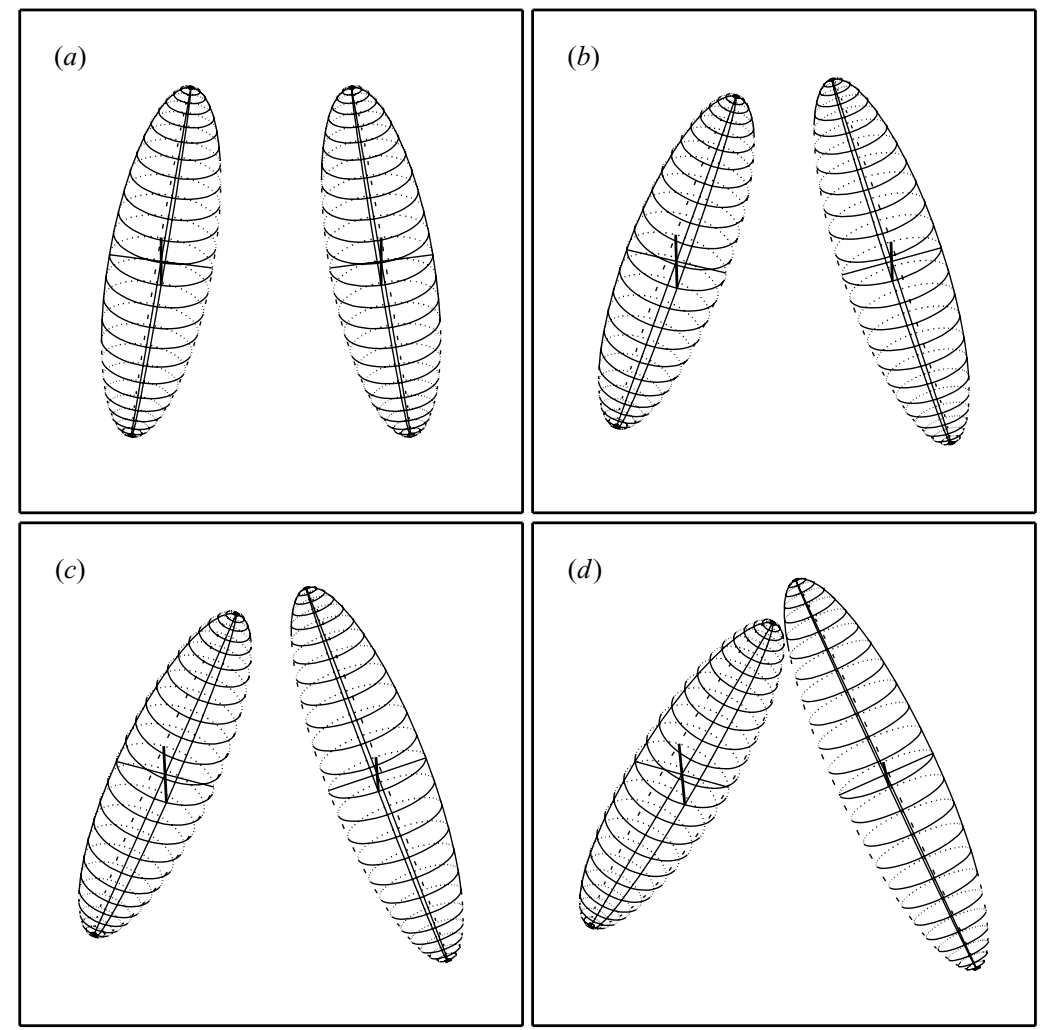

FIGURE 15. Asymmetric unstable state: the vortices have mean aspect ratios of 4.0. The volume ratio $\rho_{V}=1$ and the vertical offset $\ell^{3}=0.002\left(h_{1}+h_{2}\right)$. Times shown are $(a) t=0,(b) 305$, (c) 307, (d) 309. The initial gap is $\delta=1.01758$.

below). A similar scenario is reproduced for the symmetric case in figure 16. Here the initial gap is $\delta=1.01773$ and the growth rate of the unstable mode is $\sigma_{r}=$ 0.100516 .

These instabilities are not restricted to the ellipsoidal model, but are also observed in the full equations. We have used two simple initial configurations. In the first, we start with two spheroids, as in Dritschel (2002), with an initial horizontal offset of $\ell^{1} / r=4.38$. According to the results in Dritschel (2002), the vortices are too far apart to experience a classical merger. The critical distance for classical merger is $\ell^{1} / r=4.0$ in that case. Unlike in Dritschel (2002) we initiate the tilt instability by tilting each vortex toward the other by $1^{\circ}$. Each vortex is discretized by 80 horizontal layers. The PV is set to $4 \pi$, the time step is $\Delta t=0.025$, the dimensionless node separation is $\mu=0.075$ and the large-scale length is 0.25 , corresponding to the diameter of the vortices (see Dritschel 2002 for details of the contour dynamical (CD) numerical method). The evolution of the flow is shown in figure 17. The vortices first experience high-frequency wobbling together with lower frequency, quasi-periodic deformations. The latter deformations, not shown, correspond to a change of shape from a nearly perfect ellipsoidal shape to a crescent shape. Meanwhile, the vortices start to tilt. The tilt grows exponentially and by the time it becomes noticeable, the two vortices are collapsing into each other rapidly and the low-frequency deformations mentioned above become unimportant. The vortices then merge and intertwine progressively. 


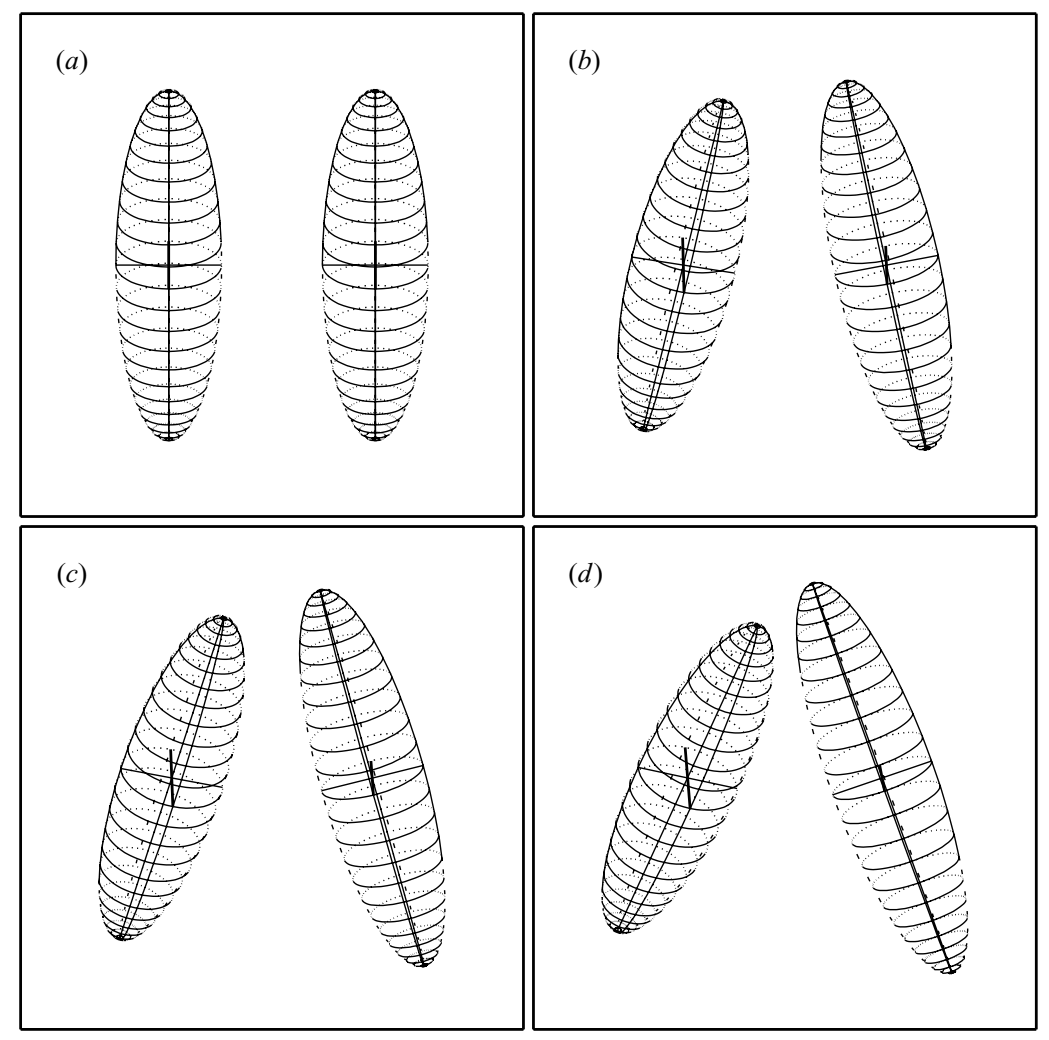

FIGURE 16. Symmetric unstable state: the vortices have mean aspect ratios of 4.0. The volume ratio $\rho_{V}=1$ and the vertical offset $\ell^{3}=0$. Times shown are $(a) t=0,(b) 312,(c) 314,(d) 316$. The initial gap is $\delta=1.01773$.

Finally the upper parts of the vortices detach and form two conically shaped satellite vortices.

In the second case, we use the marginally unstable symmetric ellipsoids, obtained using the ELM, as initial conditions. We perturb the vortices using the unstable tilting mode from the ELM stability results. The initial horizontal offset is $\ell^{1} / r=4.31$. The amplitude of the mode is set to 0.01 . Again, the PV is set to $4 \pi$, and the time step is $\Delta t=0.025$ in the $\mathrm{CD}$ simulation. The dimensionless node separation is set to 0.08 and the large-scale length is set to the mean horizontal diameter of the vortices, here 0.25 . Each vortex is now discretized by 100 horizontal layers. The evolution of the flow is shown in figure 18. The non-ellipsoidal low-frequency deformations mentioned above are again encountered here while the fast wobbling motion has virtually disappeared as a consequence of the near-equilibrium initial conditions. The quasi-periodic deformation from ellipsoidal to crescent shape is shown in the first three frames. The tilting motion then becomes evident and the vortices rapidly collapse into each other. Note that when the vortices are close enough they deform each other in a similar way to that observed in classical merger (frame $d$ ). However the tilting results in merger well away from the vortex mid-sections (above in this case because of the initial perturbation). And, as in the previous case, two small conically shaped vortices detach from the helically intertwining filaments stretching from the merged vortex. 
(a)

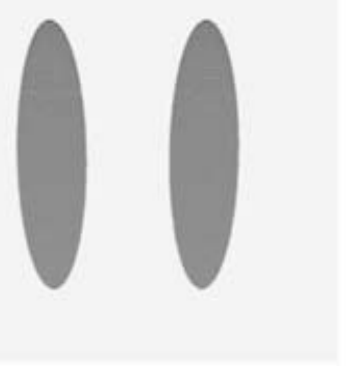

$(d)$

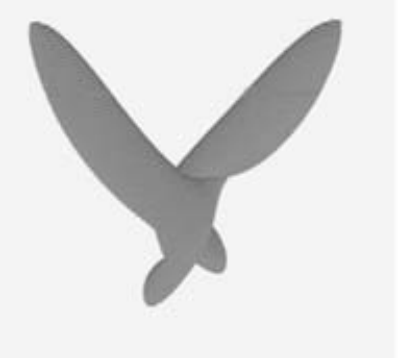

(b)

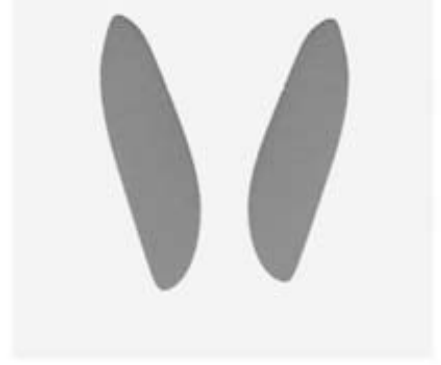

(e)

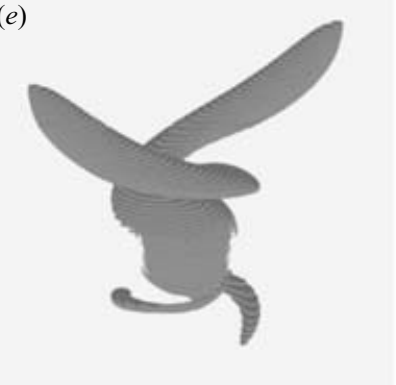

(c)
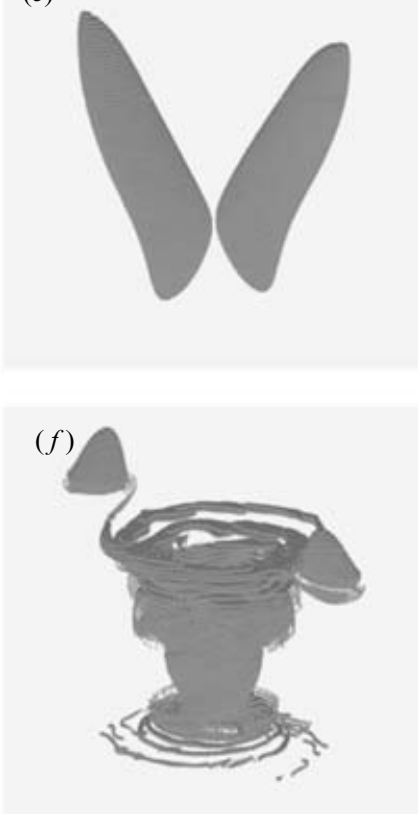

FIGURE 17. A contour dynamical simulation of the tilt instability. The times shown are (a) $t=0,(b) 60,(c)$ 64.6, (d) 65.6, (e) 67.6, and $(f)$ 76.8. The vortices have equal volume, equal $\mathrm{PV}$ and an aspect ratio of 4 . The initial condition consists of two spheroids with a horizontal offset $\ell^{1} / r=4.38$. The vortices are tilted toward one each other by $1^{\circ}$. The view is orthographic at an angle of $70^{\circ}$ from the vertical. In the first row of images, the vortices are viewed in a reference frame rotating with the vortices.

\section{Conclusions}

In this study, we have investigated the critical merger distance for oblate to moderately prolate vortices. We have considered a large parameter space spanned by the aspect ratio of each vortex, the volume ratio and the vertical offset between the two vortices. This study was enabled by the development of an accurate asymptotic model (ELM) which models vortices by ellipsoids. High-order, non-ellipsoidal deformations are disregarded in this model, but even so, this model is found to be in good agreement with the full QG equations in the prediction of the critical merger distance between two vortices.

To quantify the dependence of the critical merger distance on all of the parameters, we first found families of steadily rotating vortices parameterized by the horizontal gap between the vortices. Then, their linear stability was calculated. It was first shown that for oblate to moderately prolate vortices $(h / r \leqslant 1.6)$, the margin of stability coincides with an extremum in both the energy and the angular impulse. The instability itself is an exchange-type instability. This observation is in agreement with previous studies both in two dimensions (Dritschel 1995 and Meunier et al. 2002) and in three dimensions over a much smaller parameter space (Reinaud \& Dritschel 2002). A variety of important properties of the marginal states were then described - in particular the critical separation distance. A few general trends were pointed out, but we have been unable to find any simplifying relations applicable, even approximately, over the entire parameter space. Nonetheless, the results presented (and the database 
(a)

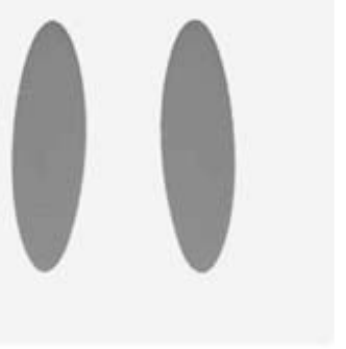

(d)

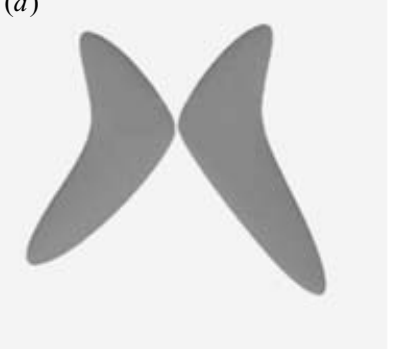

(b)

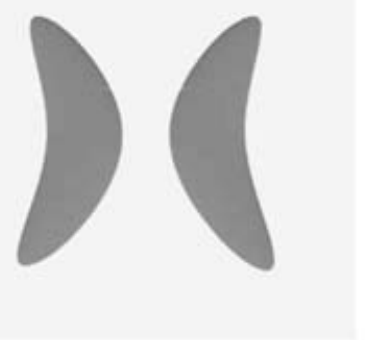

(e)

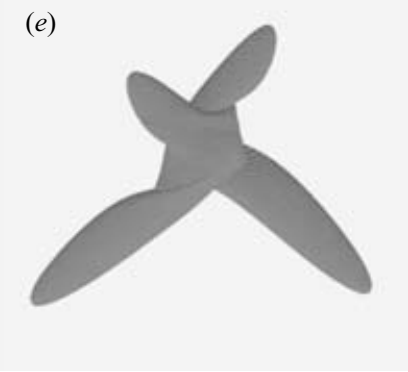

(c)
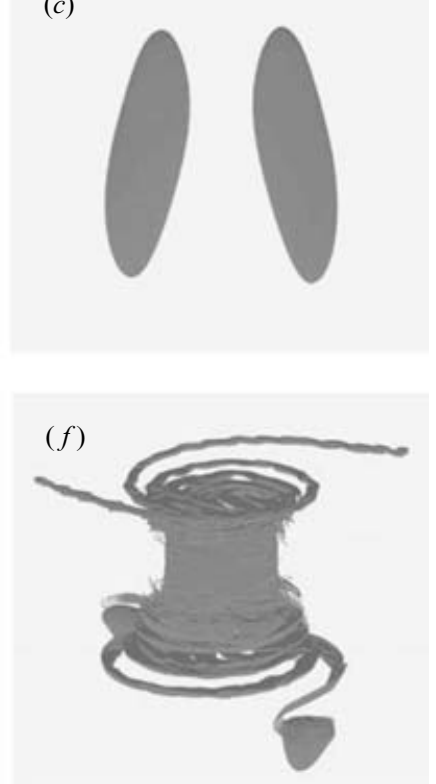

FIGURE 18. A contour dynamical simulation of the tilt instability. The times shown are (a) $t=38,(b)$ 42.5, (c) 48, (d) 52.5, (e) 53.75, and $(f)$ 63.75. The vortices have equal volume, equal PV and an aspect ratio of 4 . The initial condition consists of two marginal unstable ellipsoidal vortices. The view is orthographic at an angle of $70^{\circ}$ from the vertical. In the first row of images, the vortices are viewed in a reference frame rotating with the vortices.

from which they were extracted) give the first comprehensive picture of the conditions for the merger of two vortices in QG flows.

This investigation also led to the discovery of a new 'tilt' instability that results in the merger of prolate vortices from greater separation distances than the classical symmetric merger instability. The new tilt instability and the existence of asymmetric solutions, even for horizontally aligned vortices, significantly widens the range of known vortex interactions. The tilt instability is indeed the first instability able to precipitate the merger of prolate vortices.

This instability appears to be related to a similar instability observed for tall columns of PV between two free-slip (or isothermal) vertical boundaries in Dritschel \& de la Torre Juárez (1996), but it had never been observed for ellipsoids in an unbounded fluid. Indeed previous studies concluded that in these configurations the vortices were stable - or at least too far apart to merge. The present study demonstrates that vortices may in fact merge as a consequence of tilting toward each other.

Miyazaki et al. (1999) studied the stability of tilted isolated spheroidal vortices. The authors conclude that oblate vortices can be intrinsically unstable (for $h / r<0.62$ ). However, it seems unlikely that this instability has a strong effect on the stability of interacting vortices. Each vortex of the interacting pair is subject to an external straining flow that strongly influences its stability. The problem of strongly interacting vortices is evidently distinct from the problem of isolated vortices. The latter is of interest in its own right as it can be regarded as a model of vortex behaviour in dilute turbulence. 
An obvious next step in this research is to consider the dependence on the PV ratios between the vortices and perhaps the distribution of PV within them (considered uniform in the present study). Undoubtably, other new kinds of instability are likely to be discovered, challenging our current understanding of vortex interactions.

Support for this work has come from the UK Enegineering and Physical Sciences Research Council.

\section{REFERENCES}

Chandrasekhar, S. 1969 Ellipsoidal Figures of equilibrium, Dover.

Dritschel, D. G. 1995 A general theory for two-dimensional vortex interactions. J. Fluid Mech. 293, 269-303.

Dritschel, D. G. 2002 Vortex merger in rotating stratified flows. J. Fluid Mech. 455, 83-101.

Dritschel, D. G., Reinaud, J. N. \& McKiver, W. J. 2004 The quasi-geostrophic ellipsoidal model. J. Fluid Mech. 505, 201-223.

Dritschel, D. G. \& DE LA Torre JuÁrez, M. 1996 The instability and breakdown of tall columnar vortices in a quasi-geostrophic fluid. J. Fluid Mech. 328, 129-160.

Garrett, C. 2000 The dynamic ocean. In Perspectives in Fluid Mechanics (ed. G. K. Batchelor, H. K. Moffatt \& M.G. Worster), chap. 10, pp. 507-553. Cambridge University Press.

Gill, A. E. 1982 Atmosphere-Ocean Dynamics. Academic.

von Hardenberg, J., McWilliams, J. C., Provenzale, A., Shchpetkin, A. \& Weiss, J. B. 2000 Vortex merging in quasi-geostrohic flows. J. Fluid Mech. 412, 331-353.

Hashimoto, H., Shimonishi, T. \& MiyazaKi, T. 1999 Quasigeostrophic ellipsoidal vortices in a two-dimensional strain field. J. Phys. Soc Japan 68, 3863-3880.

Holton, J. R., Haynes, P. H., Mcintyre, M. E., Douglass, A. R., Rood, R. B. \& Pfister, L. 1995 Stratosphere-troposphere exchange. Rev. Geophys. 33, 403-439.

Hoskins, B. J., McIntyre, M. E. \& Robertson, A. W. 1985 On the use and significance of isentropic potential-vorticity maps. Q. J. R. Met. Soc. 111, 877-946.

Hua, B. L. \& Haidvogel, D. B. 1986 Numerical simulations of the vertical structure of quasigeostrophic turnulence. J. Atmos. Sci. 43, 2923-2936.

LaPlace, P. S. 1784 Théorie du Mouvement et de la Figure Elliptique des Planètes. Imprimerie de Ph.-D. Pierres, Paris.

MacLaurin, C. 1742 A Treatise on Fluxions. T., W. and T. Ruddiman, Edinburgh.

McKiver, W. J. \& Dritschel, D. G. 2003 The motion of a fluid ellipsoid in a general linear background flow. J. Fluid Mech. 474, 147-173.

Meacham, S. P. 1992 Quasigeostrophic, ellipsoidal vortices in stratified fluid. Dyn. Atmos. Oceans 16, 189-223.

Meacham, S. P., Morisson, P. J. \& Flierl, G. R. 1997 Hamiltonian moment reduction for describing vortices in shear. Phys. Fluids 9, 2310-2328.

Meacham, S. P., Pankratov, K. K., Shchepetkin, A. F. \& Zhmur, V. V. 1994 The interaction of ellipsoidal vortices with background shear flows in a stratified fluid. Dyn. Atmos. Oceans 21, $167-212$.

Meunier, P., Ehrenstein, U., Leweke, T. \& Rossi, M. 2002 A merging criterion for two-dimensional co-rotating vortices. Phys. Fluids 14, 2757-2766.

MiYazaki, T., Furuichi, Y. \& TAKahashi, N. 2001 Quasigeostrophic spheroidal vortices vortex model. J. Phys. Soc Japan 70, 1942-1953.

MiYazaki, T., Ueno, K. \& Shimonishi, T. 1999 Quasigeostrophic tilted ellipsoidal vortices. J. Phys. Soc. Japan 68, 2592-2601.

Reinaud, J. N. \& Dritschel, D. G. 2002 The merger of vertically offset quasi-geostrophic vortices. J. Fluid Mech. 469, 287-315.

Reinaud, J. N., Dritschel, D. G. \& Koudella, C. R. 2003 The shape of the vortices in quasigeostrophic turbulence. J. Fluid Mech. 474, 175-192.

Saffman, P. G. 1992 Vortex Dynamics. Cambridge University Press.

Todhunter, I. 1873 History of the Mathematical Theories of the Attraction and the Figure of the Earth. Macmillan, London. Reprinted by Dover, 1962.

Waugh, D. W. 1992 The efficiency of symmetric vortex merger. Phys. Fluids A 4, 1745-1758. 